\title{
A Systematic Review of Factors Influencing Condom Use among Female Sex Workers
}

\author{
Caitlin McLachlan ${ }^{1} \&$ Tinashe Dune $e^{1,2}$ \\ ${ }^{1}$ School of Science and Health, Western Sydney University, Parramatta, New South Wales, Australia \\ ${ }^{2}$ Translational Health Research Institute, Western Sydney University, Penrith, New South Wales, Australia \\ Correspondence: Caitlin McLachlan, School of Science and Health, Western Sydney University, Parramatta, New South \\ Wales, Australia.
}

Received: June 18, 2020

doi:10.11114/ijsss.v8i5.4902
Accepted: July 23, 2020

Available online: August 20, 2020

URL: https://doi.org/10.11114/ijsss.v8i5.4902

\begin{abstract}
A systematic review of research published in English was conducted across seven electronic databases in public health, social and psychological sciences. The aim was to ascertain the factors that influence condom use among female sex workers in the commercial sex industry globally. The perspectives and experiences of 29,112 female sex workers (FSWs) and 1,792 male clients of FSWs with regards to condom use are represented across the 47 studies included in this review. Key themes comprised of Perceived self-efficacy, Relationship Types, Condom use and Attitudes, Violence and Abuse, Location and Support, Knowledge and Perceived Risk, Perception and Perspectives of Male Clients, Drug and Alcohol use, Criminalisation and Price and Payment. Female sex workers are defined as a high-risk population for HIV/AIDS. Condoms were used less frequently in situations where structural and cultural determinants heavily impacted the experiences and decisions of FSWs. Cultural contextual factors also heavily impacted on the perspectives and perceptions of male clients and their attitudes towards condom use. Criminalization and anti-sex work legislation in many of the countries where these studies were conducted meant that FSWs were a socially marginalised and ostracized population. Recommendations are explored for theory, practice and education and future research.
\end{abstract}

Keywords: sex-work, condom, systematic review, female

\section{Introduction}

There are a range of public health issues related to low rates of condom use in sex work such as unwanted pregnancies, Human Papilloma Virus and other STIs. Taking the example of HIV/AIDS, it is apparent from the literature that female sex workers constitute a high-risk population. Despite increasing awareness and education on the transmission factors of HIV/AIDS, there is substantial literature to show that female sex workers are disproportionately affected by these infections (Wiechelt \& Shdaimah, 2015). The objective of this systematic review is to highlight and better understand the complex factors which influence condom use among females in commercial sex work, in order to improve the public health outcomes for this marginalised population.

While the term "sex work" came into use around the 1970's to define commercial sex services in exchange for material gains, sex work or prostitution has an extensive history (Panjik, 2012). Evidence of sex work can be seen in references made in early human records in 4000 B.C., with suggestions that it may have started as an ancient religious custom (Clarkson, 1939). It is difficult to estimate the number of women involved in sex work globally, as they are a hard to reach population due to stigma and marginalization (Vandepitte et al., 2006). The number of female sex workers is ever-changing with published statistics reported to be misleading due to governments' underestimating the number of FSWs as a result of restrictive views on sex work (Vandepitte et al. 2006). While there is no definitive starting point in history for sex work, we know that it is considered one of the world's oldest professions and is seen to be a constant economic feature in most societies dating back to ancient Egypt, Arabia and Israel (Clarkson, 1939). However, unlike ancient times, awareness and management of sexually transmitted infections (STIs) has increased significantly following the Human Immunodeficiency Virus/Acquired Immunodeficiency Syndrome (HIV/AIDS) epidemic in the 1980s. While sex work was thought to be a vehicle for STIs, or venereal disease as it was once called, well before the 1980s, the development of public health campaigns and programs to increase condom use in sex work multiplied in the early 1990s given that sex work was, and continues to be, considered a high-risk activity. 


\section{Literature Review}

Data from Senegal has found that female sex workers in the region are 13.5 times more likely to be living with HIV than their counterparts who do not engage in commercial sex, with a systematic review finding a 35\% prevalence of HIV among FSWs in the region (Rwema et al., 2019). Condom use has been named as the most important modifiable proximal determinant of HIV infection and transmission among female sex workers (Rwema et al., 2019).

Due to unsafe sex practices, female sex workers in Indonesia constitute one of the highest risk populations of HIV infection and transmission (Sedyaninsih-Mahamit, 1999). Similar findings can be seen in Jamaica, with an estimated 29, 690 persons living with HIV, with $14.2 \%$ of these people reporting sex with a sex worker (Bailey et al. 2018). In Swaziland, one in two women aged 25-29 are HIV positive, with younger women at a higher risk of HIV infections than their male counterparts with a $10 \%$ prevalence in young women versus a $2 \%$ prevalence in young men (Mathenjwa \& Mahara, 2012). There are many epidemiological studies which have acknowledged the low rate of consistent condom use among female sex workers in commercial and non-commercial relationships, however, they fail to provide any explanation or rather blame the low rates on failed interventions (Maher et al., 2013).

While available data provides evidence of a lack of barrier protection used among sex workers, little research has been conducted to ascertain why this occurs. While the literature shows that female sex workers are a high-risk population for HIV/STI infection, there is limited data on the structural determinants that may influence the use of barrier protection such as violence or socioeconomic status (Rwema et al., 2019). In the context of sex work, the legislative status, features of the work environment, stigma, economic factors, migration patterns, gender-based violence and community organization are all posited as key structural determinants related to HIV transmission/acquisition, however, the influence of these determinants is still poorly understood due to a lack of epidemiological data (Rwema et al., 2019). Many studies on this topic focus on individual-level constructs, which assume that not using condoms is due to behavioural choices of the individual sex-worker and this individual could assert power or control if she chose to (Urada, Morisky, Pimentel-Simbulan, Silverman \& Strathdee, 2012).

Contrastingly, some studies are now looking more in-depth at contextual factors such as social and structural factors (i.e. criminalization) that may play a more important role in condom negotiation with FSWs than originally suggested (Urada et al. 2012). Various studies have shown that low-tier (FSWs of lower socioeconomic status) sex workers engage in riskier sexual behaviours and are less likely to negotiate condom use with clients (Ma et al., 2017 \& Alam et al., 2013). In a study of 1682 low-tier FSWs in China, only 50.5\% consistently used condoms with clients, while their mid to high-tier counterparts reported more consistent rates of condom use (Ma et al., 2017). The contrast in condom use rates among the different Socio-economic status (SES) groups of FSWs highlights that there may be key behavioural and structural factors which impact condom use self-efficacy, however there is a gap in research on how these determinants influence condom use. Some of these determinants may include criminalization (Rwema et al., 2019), violence (Maher et al., 2013), support from managers or other staff (Yang et al., 2005) \& education (Kamal, Hassan \& Salikon, 2015).

Gender inequality has been named as one of the key social factors which influences the transmission of HIV, with structural analysis of HIV epidemics moving the focus of concern from personal behaviours to looking at social determinants of vulnerability (Young, Danner, Fort \& Blankenship, 2018). Constructions of gender in public health are often dichotomized into categories where health risks are based on conforming to conventional masculine and feminine roles in a cultural context (Young et al. 2018). This simplification of gender inequality does not take external structural factors into consideration, which are imperative in understanding what influences gendered sexual practices (Young et al. 2018). It has been suggested that condom use "is simply a matter of women deciding to use condoms at all times and assertively making this happen" (Gavey, McPhillips, \& Doherty, 2001, p.918). The literature suggests that condom use in commercial sexual encounters is far more complicated than this, and cannot be explained solely by women not deciding to use them. This explanation also places the onus on the FSW and disregards the male party that is also involved in transactional sex and simplifies the complexities involved in gender dynamics and sex work.

\section{Theoretical Framework}

There is much debate on whether commercial sex work is inherently exploitative to those involved or a form of empowerment by giving people autonomy over their own bodies. Either way, there is substantial literature to show that female sex workers are disproportionately affected by STI and HIV infection, despite increasing education and awareness on the transmission factors of these infections (Wiechelt \& Shdaimah, 2015).

While sex work has existed for numerous centuries, so has the stigma associated with it. In the 1800 's, a sex worker was defined as "One who openly abandons her body to a number of men, without choice, for money" (Clarkson, 1939, p. 296). Clarkson (1939) emphasizes that it is not the amount of sexual partners that a women has that makes her a "harlot", but rather the nature of her relationship with them, stating that prostitution differs from being a mistress, polygamous lover or concubine, as these women do not receive payment and therefore face less criticism and stigmatisation (Clarkson, 1939). 
The paradox of women's safety is evident here, as women are prevented from having power and money as it disrupts the patriarchal framework. If women in sex work were given more control by means of de-criminalisation or better social resources and support, evidence shows that safer sex in the sex industry would increase.

To centralize the experiences of women, feminist theory was used in this paper to interpret the findings of this review. However, feminist perspectives present two contrasting points of view with regards to commercial sex work. These debates predate the HIV epidemic and are divided into the abolitionist feminist response and the 'sex positive' feminist view (Ahmed, 2011). The abolitionist response is mainly represented by three feminists; Kathleen Barry, Catharine MacKinnon and Andrea Dworkin, who all posit that sex work is invariably exploitative and argued that the "prostituted" woman consistently acts under coercion and is subject to sexual violence (Ahmed, 2011). This model views sex work as the "oldest oppression" and an institutionalized form of sex inequality (Yingwana, 2018). Many abolitionist feminists view the criminalization of commercial sex work as a tool to rescue female sex workers from inherent entrapment, coercion and violence (Ahmed, 2011).

During the 1980's, a shift in feminist thinking argued against the abolitionist response and 'sex-positive' or third-wave feminists viewed sex work as a place of potential agency for women, as opposed to subordination and coercion as suggested by abolitionist feminists (Ahmed, 2011). This model of feminism saw the selling of sex as an occupation like any other (Yingwana, 2018). The new feminist frame of sex work rejected the view of female sex workers as exploited slaves and allowed sex-positive feminists to become allies of female sex workers, creating new alliances and a new dynamic and allowing feminists and sex workers to work together and push for the decriminalization of sex work in order to improve the rights and safety of FSWs (Ahmed, 2011).

These frameworks dichotomize female sex workers as either a victim or an agent (Choi \& Holroyd, 2007), however this paper aims to acknowledge the complexities faced by FSWs that can influence decision-making rather than categorizing them. By looking at these findings through a feminist, sex positive lens, this review aimed to identify the multidimensional structural, social and cultural factors related to condom use in commercial sex work settings.

\section{Methods}

In line with Preferred Reporting Items for Systematic Reviews and Meta-Analyses (PRISMA) guidelines (Moher, Liberati, Tetzlaff, Altmann \& Group, 2009), a systematic review using narrative analysis was conducted to identify research that investigated factors influencing condom use among female sex workers.

\section{Search Strategy}

Evidence was searched for between June and August 2019 and additional searches were conducted periodically until November 2019 in order to identify any new studies published.

Primary peer-reviewed articles were sought from seven major public health and social and psychological science electronic databases: ProQuest Central, CINAHL Plus, PsycInfo, Medline, Pubmed, Cochrane and Psychology database. A preliminary search of ProQuest Central was undertaken by the both authors with the goal of identifying the key words contained in the study titles and abstracts. Appropriate key words were discussed, elaborated and refined. A second search, using all identified keywords was conducted across the seven databases listed above. The search string used to locate articles was ti(sex work OR prostitut* OR sex worker) AND ti(women OR woman OR female OR cis-gender NOT transgender) AND ti(condom OR condom use OR barrier protection OR prophylactic OR safer sex OR safe sex) AND ab(self-efficacy OR agency OR negotiat* OR personal efficacy). Details of the search strategy, including the search terms and combinations are summarised in Table 1. 
Table 1. Summary of the inclusion/exclusion criteria and keywords

\begin{tabular}{|c|c|c|c|}
\hline Parameters & Inclusion & Exclusion & Key words/steps \\
\hline Location & International & None & N/A \\
\hline Language & Written in English & Other languages & Select for English only \\
\hline Time & Any & None & N/A \\
\hline Population & $\begin{array}{l}\text { Literature which includes } \\
\text { cis-gender female sex workers, } \\
\text { their clients and condom use }\end{array}$ & $\begin{array}{l}\text { Literature which does not } \\
\text { focus on cis-gender female } \\
\text { sex workers, their clients and } \\
\text { condom use }\end{array}$ & $\begin{array}{l}\text { (Title) sex work OR } \\
\text { Prostitut* OR } \\
\text { Sex worker } \\
\text { AND } \\
\text { (Title) women OR woman OR } \\
\text { female OR cis-gender NOT } \\
\text { transgender }\end{array}$ \\
\hline Phenomena/Target & $\begin{array}{l}\text { Studies concerned with the use } \\
\text { of condoms in a commercial } \\
\text { sex setting involving a female } \\
\text { sex worker }\end{array}$ & $\begin{array}{l}\text { Studies not concerned with } \\
\text { the use of condoms in a } \\
\text { commercial sex setting } \\
\text { involving a female sex } \\
\text { worker }\end{array}$ & $\begin{array}{l}\text { AND (Title) condom OR condom } \\
\text { use OR barrier protection OR } \\
\text { prophylactic OR safer sex OR safe } \\
\text { sex } \\
\text { AND } \\
\text { (Abstract) self-efficacy OR agency } \\
\text { OR personal efficacy OR negotiat* }\end{array}$ \\
\hline Study/literature type & $\begin{array}{l}\text { Published peer-reviewed } \\
\text { primary research including } \\
\text { qualitative, quantitative and } \\
\text { mixed method designs }\end{array}$ & $\begin{array}{l}\text { Published literature which } \\
\text { DO NOT include qualitative, } \\
\text { quantitative and mixed } \\
\text { methods of data collection } \\
\text { and analysis }\end{array}$ & N/A \\
\hline
\end{tabular}

\section{Inclusion and Exclusion Criteria}

Both qualitative and quantitative data from mixed-method studies that reported on factors influencing condom use among female sex workers until August 2019 were included. Studies conducted on or with female sex workers or their respective male clients were included. No age restriction was applied to the exclusion criteria for FSWs as the age of consent differed according to region, in addition to a lack of legislation not controlling for FSWs who were underage at the time of the study. Excluding the experiences of underage FSWs would limit the voices of women in sex work. We decided to exclude studies involving transgender women as their lived experiences may differ vastly from cis-gender female sex workers and this topic is comprised of its own complexities which should be addressed in greater detail in another systematic review.

\section{Data Synthesis}

This systematic review analysed the literature using a thematic approach developed by Thomas and Harden (Thomas \& Harden, 2008) that extracted, synthesised, analysed and interpreted the findings of the included literature. Three steps were followed: 1) line by line coding of results, discussion and conclusion sections of the primary studies: 2) development of descriptive themes; and 3) generation of analytical themes towards a synthesised presentation of results. The first author completed a preliminary synthesis of primary data followed by a review and disagreement resolution by the second author.

\section{Quality Assessment}

In order to assess the quality of the quantitative papers included in this systematic review, we used the Effective Public Health Practice Project's Quality Assessment Tool (QAT) for Quantitative Studies (Thomas, 2003). The QAT was used to appraise 40 of the 47 studies included, those that employed quantitative or mixed-methods approaches to collecting, analyzing and interpreting the data. Studies that only used qualitative methods were not evaluated using the QAT. The first author assessed the quality of the studies with the second author reviewing the article assessments.

\section{Results}

From the 153 potentially relevant articles identified, 47 articles were included in the systematic review (S1 Fig). 
Total number of literature identified from the seven

databases $(n=153)$

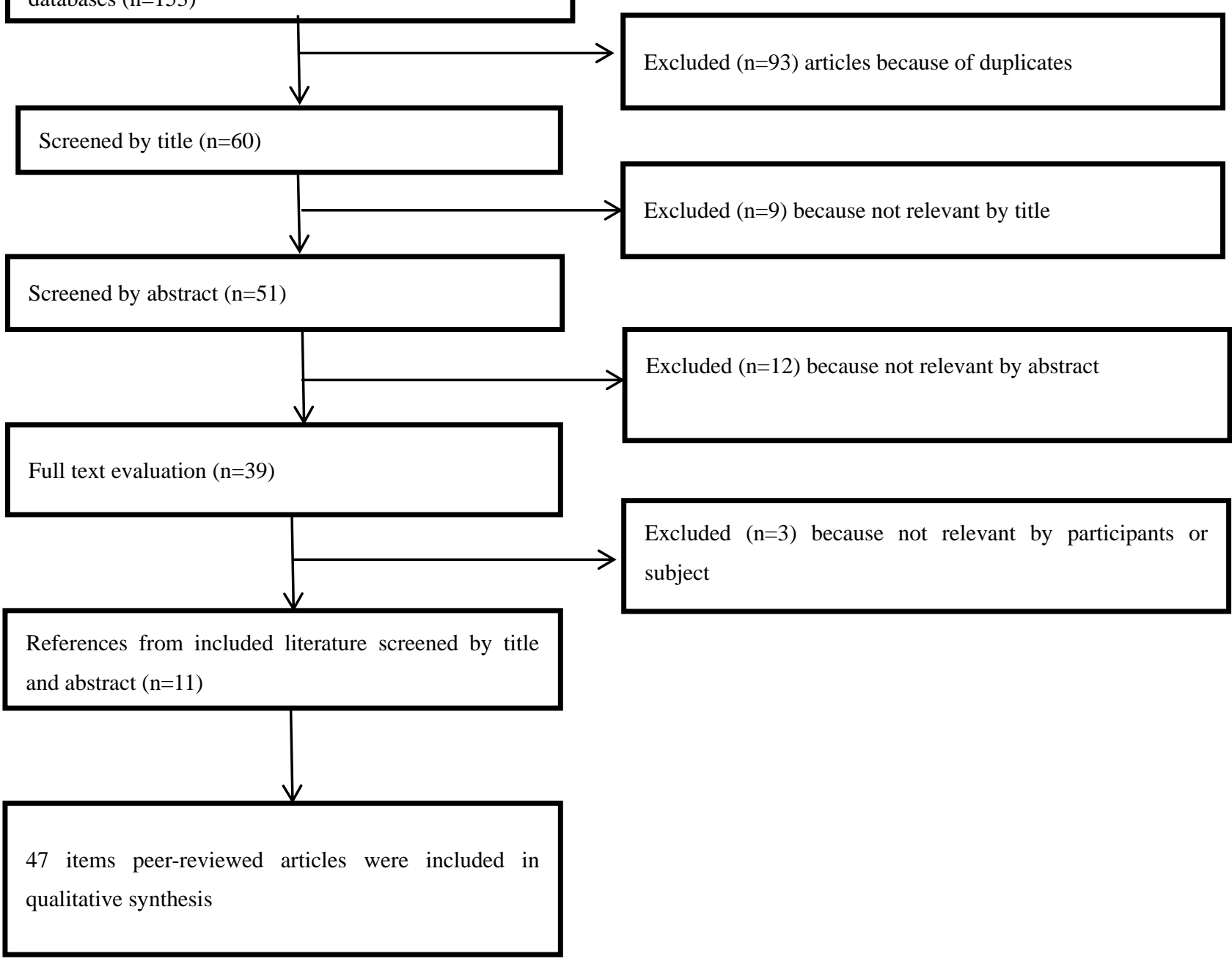

S1 Fig. Article Selection Process

\section{Sample}

The characteristics of each study are summarised in Table 2. The perspectives of FSWs and clients of FSWs with regards to factors affecting condom use in a commercial sex setting were synthesised and presented below. The perspectives of approximately 29,112 female sex workers, 1,792 clients of female sex workers, 6 facilitators of sex work and two main partners of sex workers are presented below. The majority of the research was conducted in China (10), with the remainder being conducted in Indonesia (4), India (4), Cambodia (3), Dominican Republic (2), Bangladesh (2), Singapore (2), Mexico (2), Jamaica (1), Papua New Guinea (1), Hong Kong (1), Scotland (1), Armenia (1), USA (1), Zimbabwe (1), Nepal (1), Vietnam (1), The Gambia (1), South Korea (1), Montenegro (1), Korea (1), Swaziland (1), Kenya (1), Senegal (1), Canada (1), and the Philippines (1). Three papers included the perspectives of male clients in addition to female sex workers, five papers focused solely on the perspectives of male clients and thirty-six papers specified locations of commercial sex transactions, with the most common locations being a combination of brothels and commercial entertainment establishments (i.e., clubs, bars and massage parlours).

The age range of FSWs differed in each study, however the ages of FSWs in this systematic review ranged from 13-72 years of age. Various factors in relation to condom use were touched on in the papers and some of these included self-efficacy, negotiation skills, type of relationship with client, condom attitudes, sexual health knowledge, criminalisation and perceived risk. These will be discussed in further detail in the results section. 


\section{Research foci and Theoretical Approach}

The included studies primarily focused on evaluating the self-efficacy of female sex workers in regards to condom use and HIV/STI risk behaviour. A number of studies also explored condom use self-efficacy with regards to male clients of female sex workers. All studies aimed to make recommendations for future interventions to promote safe sex among female sex workers or their clients.

Twenty-one papers explicitly indicated the use of a theoretical approach to guide the research. Among those that did were Grounded Theory, Feminist Framework, Social Cognitive theory, Information Motivation Behavioural Skills model, Health Belief model, Theory of Gender and Power, Modified Social Ecological Model, Theory of Planned Behaviour, Symbolic Interactionism, AIDS Risk Reduction model, Theory of Self-Efficacy, Risk Environment framework, Structural interventions framework and Greens' PRECDE-PROCEED framework.

\section{Research Design and Methodology}

Quantitative methodology using surveys were the most common data collection strategy. Seven studies used qualitative approaches, with interviews being the most common method. Two used a mixed-methods approach. Given the emphasis on quantitative methods, a variety of statistical analyses were applied including descriptive statistics, multiple regression and hierarchical regression. Qualitative studies undertook thematic or content analyses.

Table 2. Characteristics of 47 Studies Included in the Systematic Review

\begin{tabular}{|c|c|c|c|c|c|c|c|c|}
\hline Author/Year & $\begin{array}{l}\text { Literatu } \\
\text { re Type }\end{array}$ & $\begin{array}{l}\text { Populati } \\
\text { on }\end{array}$ & Country & $\begin{array}{l}\text { Commercial } \\
\text { Setting }\end{array}$ & Sample Size & $\begin{array}{l}\text { Design/ data } \\
\text { collection }\end{array}$ & $\begin{array}{l}\text { Analytical } \\
\text { Approach }\end{array}$ & Theoretical Framework \\
\hline $\begin{array}{l}\text { Alam, N. et al. } \\
\text { (2013) }\end{array}$ & $\begin{array}{l}\text { Journal } \\
\text { Article }\end{array}$ & $\begin{array}{l}\text { Female } \\
\text { sex } \\
\text { workers }\end{array}$ & $\begin{array}{l}\text { Banglade } \\
\text { sh }\end{array}$ & $\begin{array}{l}\text { Brothels, hotels } \\
\text { \& street-based }\end{array}$ & 1395 FSWs & $\begin{array}{l}\text { Quantitative: } \\
\text { Structured } \\
\text { questionnaire }\end{array}$ & $\begin{array}{l}\text { Chi-square tests, } \\
\text { t-tests, bivariate } \\
\text { and multivariate } \\
\text { cumulative logit } \\
\text { models }\end{array}$ & N/A \\
\hline $\begin{array}{l}\text { Bailey, A. et al. } \\
\text { (2018) }\end{array}$ & $\begin{array}{l}\text { Journal } \\
\text { Article }\end{array}$ & $\begin{array}{l}\text { Female } \\
\text { sex } \\
\text { workers, } \\
\text { Male } \\
\text { clients of } \\
\text { FSWs, } \\
\text { Main } \\
\text { partners } \\
\text { \& } \\
\text { facilitato } \\
\text { rs of sex } \\
\text { work }\end{array}$ & Jamaica & N/A & $\begin{array}{l}28 \text { FSWs, } 8 \text { male } \\
\text { clients of FSWs, } \\
2 \text { main partners of } \\
\text { FSWs, } \\
\text { facilitators of sex } \\
\text { work }\end{array}$ & $\begin{array}{l}\text { Qualitative: } \\
\text { Semi-structured } \\
\text { in-depth interviews }\end{array}$ & Thematic analysis & Grounded Theory \\
\hline $\begin{array}{l}\text { Barrington, C. et } \\
\text { al. (2009) }\end{array}$ & $\begin{array}{l}\text { Journal } \\
\text { Article }\end{array}$ & $\begin{array}{l}\text { Male } \\
\text { clients of } \\
\text { FSWs }\end{array}$ & $\begin{array}{l}\text { Dominica } \\
\mathrm{n} \\
\text { Republic }\end{array}$ & Brothel based & $\begin{array}{l}318 \text { male clients } \\
\text { of FSWs }\end{array}$ & $\begin{array}{l}\text { Quantitative: } \\
\text { Survey }\end{array}$ & $\begin{array}{l}\text { Descriptive } \\
\text { statistics, } \\
\text { Bivariate and } \\
\text { Multivariate } \\
\text { logistic regression }\end{array}$ & N/A \\
\hline $\begin{array}{l}\text { Bharat, S. et al. } \\
\text { (2013) }\end{array}$ & $\begin{array}{l}\text { Journal } \\
\text { Article }\end{array}$ & $\begin{array}{l}\text { Female } \\
\text { sex } \\
\text { workers }\end{array}$ & India & $\begin{array}{l}\text { Varied: Brothel } \\
\text { and non-brothel }\end{array}$ & 5,498 FSWs & $\begin{array}{l}\text { Quantitative: } \\
\text { Survey }\end{array}$ & $\begin{array}{l}\text { Percentages and } \\
\text { summary } \\
\text { measures, two } \\
\text { separate logistic }\end{array}$ & N/A \\
\hline
\end{tabular}




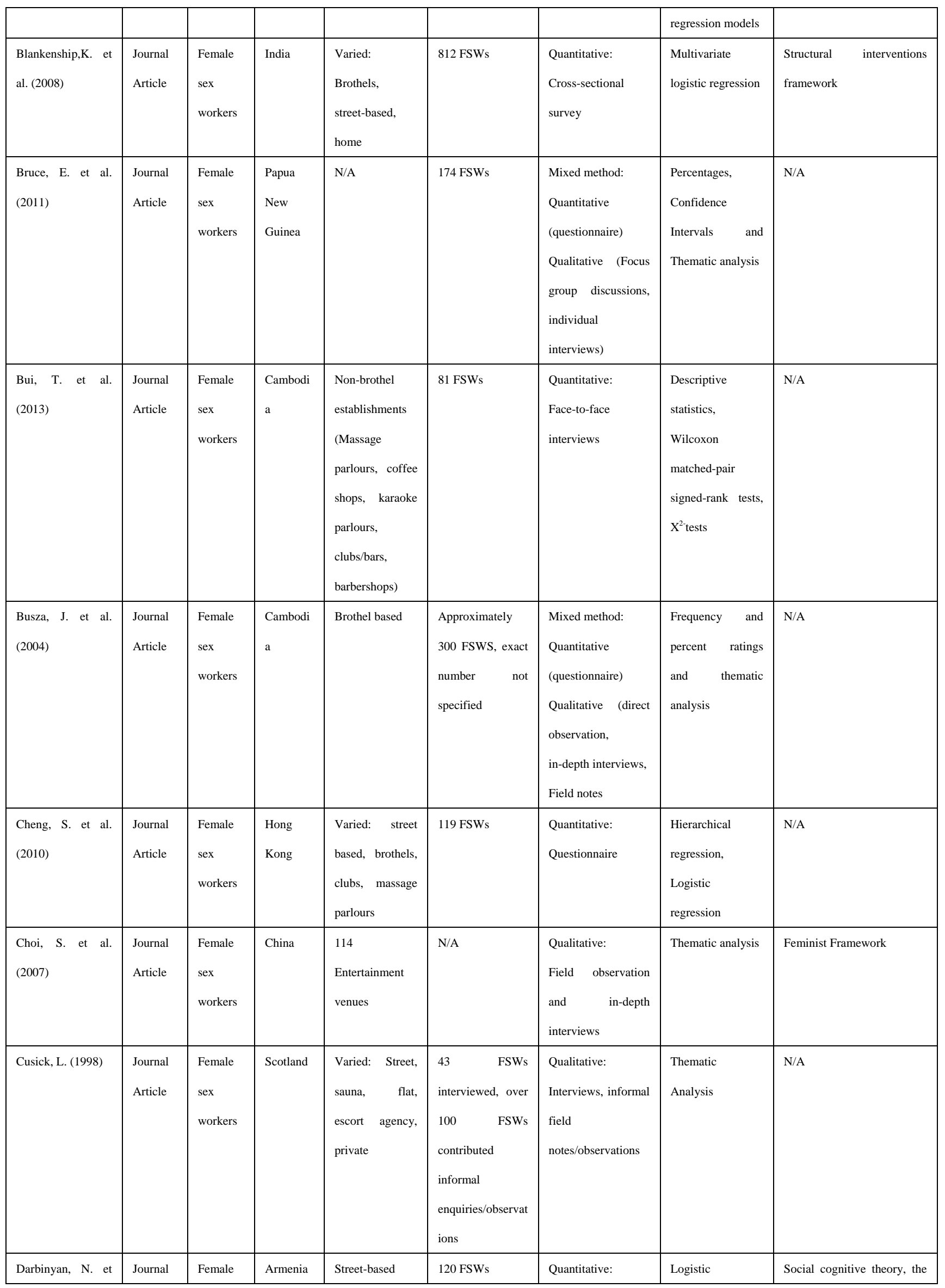




\begin{tabular}{|c|c|c|c|c|c|c|c|c|}
\hline al. (2011) & Article & $\begin{array}{l}\text { sex } \\
\text { workers }\end{array}$ & & & & $\begin{array}{l}\text { Interview-administe } \\
\text { red questionnaire }\end{array}$ & $\begin{array}{l}\text { regression } \\
\text { analysis }\end{array}$ & $\begin{array}{l}\text { Information-Motivation } \\
\text { Behavioral skills model, the } \\
\text { Health Belief Model \& the } \\
\text { Theory of Gender \& Power }\end{array}$ \\
\hline $\begin{array}{l}\text { Decker, M. et al. } \\
\text { (2019) }\end{array}$ & $\begin{array}{l}\text { Journal } \\
\text { Article }\end{array}$ & $\begin{array}{l}\text { Female } \\
\text { sex } \\
\text { workers }\end{array}$ & USA & Street-based & $250 \mathrm{FSWs}$ & $\begin{array}{l}\text { Quantitative: } \\
\text { Interviewer-adminis } \\
\text { tered computer } \\
\text { assisted personal } \\
\text { interview (CAPI) }\end{array}$ & $\begin{array}{l}\text { Chi-square tests, } \\
\text { Mann-Whitney U } \\
\text { test, multivariate } \\
\text { logistic regression } \\
\text { analyses }\end{array}$ & N/A \\
\hline $\begin{array}{l}\text { Elmes, J. et al. } \\
\text { (2014) }\end{array}$ & $\begin{array}{l}\text { Journal } \\
\text { Article }\end{array}$ & $\begin{array}{l}\text { Female } \\
\text { sex } \\
\text { workers }\end{array}$ & $\begin{array}{l}\text { Zimbabw } \\
\text { e }\end{array}$ & N/A & 311 FSWs & $\begin{array}{l}\text { Quantitative: } \\
\text { Questionnaire }\end{array}$ & $\begin{array}{l}\text { Wilcoxon } \\
\text { rank-sum tests, } \\
\text { Fisher's exact test, } \\
\text { Multivariate } \\
\text { regression } \\
\text { analysis }\end{array}$ & N/A \\
\hline $\begin{array}{l}\text { Fauk, N. et al. } \\
\text { (2018) }\end{array}$ & $\begin{array}{l}\text { Journal } \\
\text { Article }\end{array}$ & $\begin{array}{l}\text { Male } \\
\text { clients of } \\
\text { FSWs }\end{array}$ & Indonesia & N/A & $\begin{array}{l}42 \text { male clients of } \\
\text { FSWs }\end{array}$ & $\begin{array}{l}\text { Qualitative: } \\
\text { Face-to-face } \\
\text { interviews }\end{array}$ & Thematic analysis & The Health Belief Model \\
\hline $\begin{array}{l}\text { Ford, K. et al. } \\
(2000)\end{array}$ & $\begin{array}{l}\text { Journal } \\
\text { Article }\end{array}$ & $\begin{array}{l}\text { Female } \\
\text { sex } \\
\text { workers }\end{array}$ & Indonesia & $\begin{array}{l}\text { Low-tier } \\
\text { brothels }\end{array}$ & $\begin{array}{l}407 \text { FSWs (1994) } \\
300 \text { FSWs (1996) } \\
631 \text { FSWs (1998) }\end{array}$ & $\begin{array}{l}\text { Quantitative: } \\
\text { Cross-sectional } \\
\text { surveys/face-to-face } \\
\text { interviews }\end{array}$ & $\begin{array}{l}\text { T-tests, multiple } \\
\text { regression } \\
\text { analysis, logistic } \\
\text { regression } \\
\text { analysis }\end{array}$ & $\begin{array}{l}\text { The Health Belief Model \& } \\
\text { Social cognitive theory }\end{array}$ \\
\hline $\begin{array}{l}\text { Ghimire, L. et al. } \\
\text { (2011) }\end{array}$ & $\begin{array}{l}\text { Journal } \\
\text { Article }\end{array}$ & $\begin{array}{l}\text { Female } \\
\text { sex } \\
\text { workers }\end{array}$ & Nepal & N/A & 15 FSWs & $\begin{array}{l}\text { Qualitative: } \\
\text { In-depth interviews, } \\
\text { field observation }\end{array}$ & Thematic analysis & N/A \\
\hline $\begin{array}{l}\text { Grayman, J. et al. } \\
(2005)\end{array}$ & $\begin{array}{l}\text { Journal } \\
\text { Article }\end{array}$ & $\begin{array}{l}\text { Female } \\
\text { sex } \\
\text { workers }\end{array}$ & Vietnam & $\begin{array}{l}\text { Varied: } \\
\text { Brothels, bars, } \\
\text { restaurants }\end{array}$ & $610 \mathrm{FSWs}$ & $\begin{array}{l}\text { Quantitative: } \\
\text { Face-to-face } \\
\text { interviews }\end{array}$ & $\begin{array}{l}\text { Logistic } \\
\text { regression } \\
\text { analyses }\end{array}$ & N/A \\
\hline $\begin{array}{l}\text { Grosso, A. et al. } \\
\text { (2015) }\end{array}$ & $\begin{array}{l}\text { Journal } \\
\text { Article }\end{array}$ & $\begin{array}{l}\text { Female } \\
\text { sex } \\
\text { workers }\end{array}$ & $\begin{array}{l}\text { The } \\
\text { Gambia }\end{array}$ & N/A & 248 FSWs & $\begin{array}{l}\text { Quantitative: } \\
\text { Surveys }\end{array}$ & $\begin{array}{l}\text { Descriptive } \\
\text { statistics, } \\
\text { Bivariate and } \\
\text { Multivariate } \\
\text { logistic regression }\end{array}$ & $\begin{array}{l}\text { Modified Social Ecological } \\
\text { Model (MSEM) }\end{array}$ \\
\hline Jung, M. (2013) & $\begin{array}{l}\text { Journal } \\
\text { Article }\end{array}$ & $\begin{array}{l}\text { Female } \\
\text { sex } \\
\text { workers } \\
\text { and male } \\
\text { clients }\end{array}$ & $\begin{array}{l}\text { South } \\
\text { Korea }\end{array}$ & $\begin{array}{l}\text { Red light } \\
\text { districts (groups } \\
\text { of } 10-50 \\
\text { brothels) }\end{array}$ & $\begin{array}{l}\text { 1,083 FSWs, } 118 \\
\text { Johns (male } \\
\text { clients) }\end{array}$ & $\begin{array}{l}\text { Quantitative: } \\
\text { Surveys }\end{array}$ & $\begin{array}{ll}\text { Percentage } & \text { and } \\
\text { frequency } & \text { rates, } \\
\text { Ordinary } & \text { least } \\
\text { squares } & \text { (OLS) } \\
\text { regression } & \\
\text { analyses } & \end{array}$ & Theory of Planned Behavior \\
\hline $\begin{array}{l}\text { Kamal, M. et al. } \\
\text { (2015) }\end{array}$ & $\begin{array}{l}\text { Journal } \\
\text { Article }\end{array}$ & FSWs & $\begin{array}{l}\text { Banglade } \\
\text { sh }\end{array}$ & $\begin{array}{l}\text { Varied: } \\
\text { Street-based, } \\
\text { homes, hotels }\end{array}$ & 484 FSWs & $\begin{array}{l}\text { Quantitative: } \\
\text { Cross-sectional } \\
\text { surveys, interviews }\end{array}$ & $\begin{array}{l}\text { Bivariate/multivar } \\
\text { iable binary } \\
\text { logistic regression }\end{array}$ & N/A \\
\hline
\end{tabular}




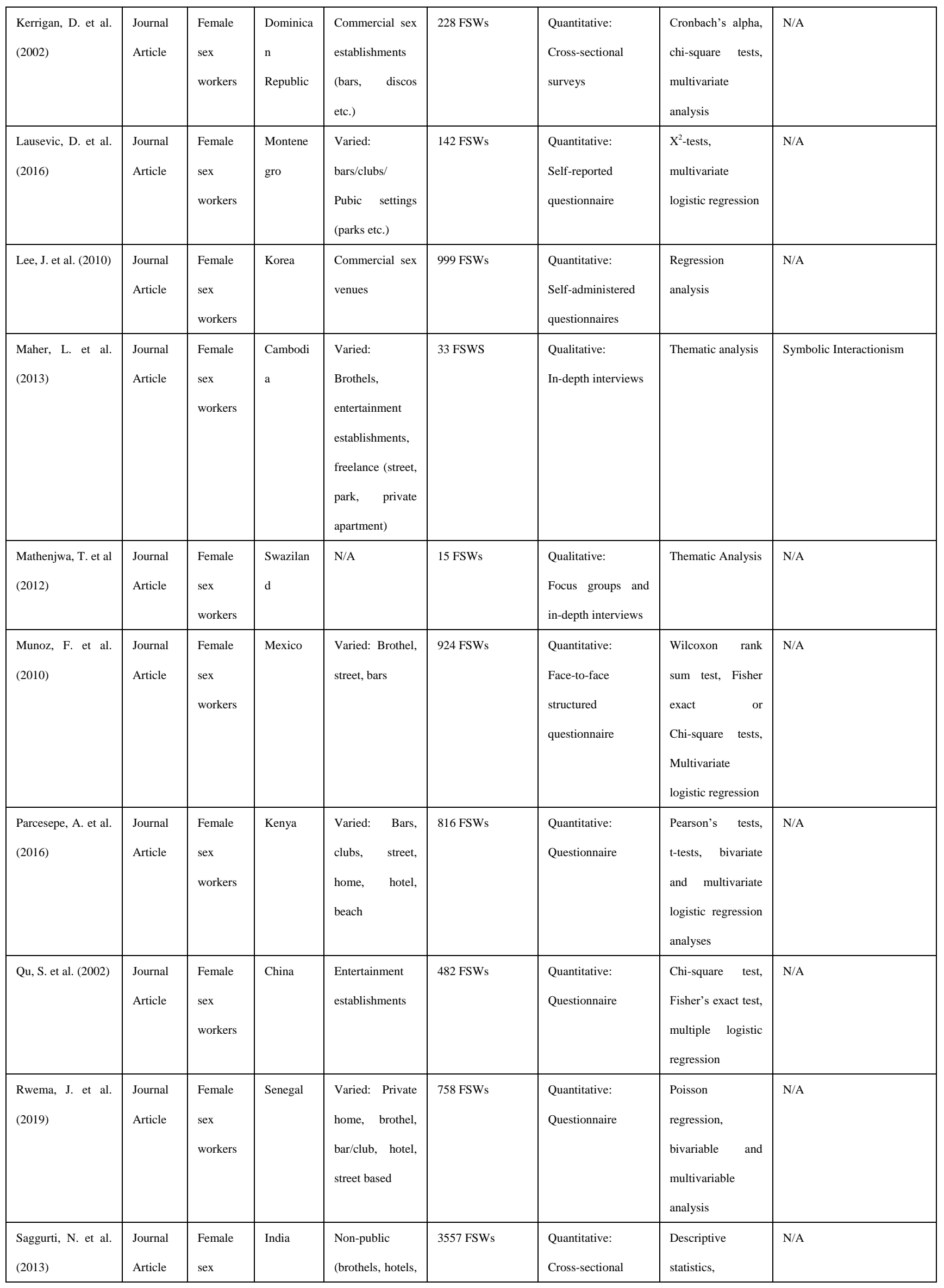




\begin{tabular}{|c|c|c|c|c|c|c|c|c|}
\hline & & workers & & $\begin{array}{l}\text { cafes, homes) } \\
\text { Public (streets, } \\
\text { market areas, } \\
\text { highways, } \\
\text { cinemas) }\end{array}$ & & survey & Bivariate analysis & \\
\hline $\begin{array}{l}\text { Sedyaningsih-Mah } \\
\text { amit, E. et al. } \\
\text { (1999) }\end{array}$ & $\begin{array}{l}\text { Journal } \\
\text { Article }\end{array}$ & $\begin{array}{l}\text { Female } \\
\text { sex } \\
\text { workers }\end{array}$ & Indonesia & Brothel complex & 459 FSWs & $\begin{array}{l}\text { Quantitative: } \\
\text { Surveys }\end{array}$ & $\begin{array}{l}\text { Univariate, } \\
\text { bivariate \& } \\
\text { multivariate } \\
\text { analyses, logistic } \\
\text { regression, } \\
\text { Spearman } \\
\text { correlation } \\
\text { coefficients }\end{array}$ & $\begin{array}{l}\text { AIDS risk reduction model, } \\
\text { Health belief model and } \\
\text { Theory of self-efficacy }\end{array}$ \\
\hline $\begin{array}{l}\text { Shannon, K. et al. } \\
\text { (2009) }\end{array}$ & $\begin{array}{l}\text { Journal } \\
\text { Article }\end{array}$ & $\begin{array}{l}\text { Female } \\
\text { sex } \\
\text { workers }\end{array}$ & Canada & Street-based & 205 FSWs & $\begin{array}{l}\text { Quantitative: } \\
\text { Semi-structured } \\
\text { questionnaire }\end{array}$ & $\begin{array}{l}\text { Pearson test, } \\
\text { t-tests, } \\
\text { Mann-Whitney U } \\
\text { test, multivariate } \\
\text { logistic regression }\end{array}$ & $\begin{array}{ll}\text { Risk } & \text { Environment } \\
\text { Framework } & \end{array}$ \\
\hline $\begin{array}{l}\text { Thorpe, L. et al. } \\
\text { (1997) }\end{array}$ & $\begin{array}{l}\text { Journal } \\
\text { Article }\end{array}$ & $\begin{array}{l}\text { Female } \\
\text { sex } \\
\text { workers } \\
\text { and male } \\
\text { tourist } \\
\text { clients }\end{array}$ & Indonesia & $\begin{array}{l}\text { Varied: Private } \\
\text { rented } \\
\text { rooms/freelance }\end{array}$ & $\begin{array}{l}80 \text { FSWs, } 100 \\
\text { tourist clients }\end{array}$ & $\begin{array}{l}\text { Quantitative: } \\
\text { Survey }\end{array}$ & $\begin{array}{l}\text { Odds ratios with } \\
95 \% \quad \text { CI, } \\
\text { univariate } \\
\text { statistical } \\
\text { methods, Logistic } \\
\text { regression }\end{array}$ & $\begin{array}{l}\text { Health Belief Model, } \\
\text { Bandura's theory of } \\
\text { self-efficacy }\end{array}$ \\
\hline $\begin{array}{l}\text { Urada, L. et al. } \\
\text { (2012) }\end{array}$ & $\begin{array}{l}\text { Journal } \\
\text { Article }\end{array}$ & $\begin{array}{l}\text { Female } \\
\text { sex } \\
\text { workers }\end{array}$ & $\begin{array}{l}\text { The } \\
\text { Philippin } \\
\text { es }\end{array}$ & Bars/Spas & $498 \mathrm{FSWs}$ & $\begin{array}{l}\text { Quantitative: } \\
\text { Interview-led } \\
\text { surveys }\end{array}$ & $\begin{array}{l}\text { t-tests, Pearson's } \\
\text { chi-square test, } \\
\text { univariate and } \\
\text { multivariate } \\
\text { logistic regression, } \\
\text { multilevel } \\
\text { modeling }\end{array}$ & $\begin{array}{ll}\text { Risk Environment } \\
\text { Framework }\end{array}$ \\
\hline $\begin{array}{l}\text { Vejella, S. et al } \\
\text { (2016) }\end{array}$ & $\begin{array}{l}\text { Journal } \\
\text { Article }\end{array}$ & $\begin{array}{l}\text { Female } \\
\text { sex } \\
\text { workers }\end{array}$ & India & $\begin{array}{l}\text { Non-public } \\
\text { (brothels, hotels, } \\
\text { cafes, homes) } \\
\text { Public (street, } \\
\text { market areas, } \\
\text { highways, } \\
\text { cinemas) }\end{array}$ & $\begin{array}{ll}1986 & \text { FSWs } \\
(2010) & \\
1973 & \text { FSWs } \\
(2012) & \end{array}$ & $\begin{array}{l}\text { Quantitative: } \\
\text { Two round of } \\
\text { cross-sectional } \\
\text { surveys }\end{array}$ & $\begin{array}{l}\text { Descriptive } \\
\text { statistics, bivariate } \\
\text { analysis, multiple } \\
\text { logistic regression } \\
\text { analysis }\end{array}$ & $\begin{array}{l}\text { Bandura's Theory of } \\
\text { Self-Efficacy }\end{array}$ \\
\hline $\begin{array}{l}\text { Volkmann, T. et al. } \\
\text { (2014) }\end{array}$ & $\begin{array}{l}\text { Journal } \\
\text { Article }\end{array}$ & $\begin{array}{l}\text { Male } \\
\text { clients of } \\
\text { FSWs }\end{array}$ & Mexico & N/A & 393 male clients & $\begin{array}{l}\text { Quantitative: } \\
\text { Questionnaire, } \\
\text { Interviews }\end{array}$ & $\begin{array}{l}\text { Hierarchical } \\
\text { multiple } \\
\text { regression } \\
\text { analysis }\end{array}$ & Social Cognitive Theory \\
\hline
\end{tabular}




\begin{tabular}{|c|c|c|c|c|c|c|c|c|}
\hline (2009) & Article & $\begin{array}{l}\text { sex } \\
\text { workers }\end{array}$ & & based & & Surveys & $\begin{array}{l}\text { statistics, t-tests, } \\
\text { Pearson } \\
\text { correlations, path } \\
\text { analysis }\end{array}$ & \& Social Cognitive Theory \\
\hline $\begin{array}{l}\text { Wee, S. et al. } \\
\text { (2004) }\end{array}$ & $\begin{array}{l}\text { Journal } \\
\text { Article }\end{array}$ & $\begin{array}{l}\text { Male } \\
\text { clients of } \\
\text { FSWs }\end{array}$ & $\begin{array}{l}\text { Singapor } \\
\mathrm{e}\end{array}$ & N/A & $\begin{array}{l}229 \text { male clients } \\
\text { of FSWs }\end{array}$ & $\begin{array}{l}\text { Quantitative: } \\
\text { Questionnaire }\end{array}$ & $\begin{array}{l}\text { Pearson } \\
\text { correlations, } \\
\text { t-tests, } \\
\text { Mann-Whitney U } \\
\text { test, binary } \\
\text { logistic regression }\end{array}$ & N/A \\
\hline $\begin{array}{l}\text { Wong, M. et al. } \\
\text { (1999) }\end{array}$ & $\begin{array}{l}\text { Journal } \\
\text { Article }\end{array}$ & $\begin{array}{l}\text { Female } \\
\text { sex } \\
\text { workers }\end{array}$ & $\begin{array}{l}\text { Singapor } \\
\mathrm{e}\end{array}$ & Brothel based & 225 FSWs & $\begin{array}{l}\text { Quantitative: } \\
\text { Questionnaire }\end{array}$ & $\begin{array}{l}\text { Chi-squared, } \\
\text { t-tests, } \\
\text { Multivariate } \\
\text { regression } \\
\text { analysis }\end{array}$ & $\begin{array}{l}\text { Green's } \\
\text { PRECEDE-PROCEED } \\
\text { framework }\end{array}$ \\
\hline $\begin{array}{l}\text { Yang, H. et al. } \\
\text { (2005) }\end{array}$ & $\begin{array}{l}\text { Journal } \\
\text { Article }\end{array}$ & $\begin{array}{l}\text { Female } \\
\text { sex } \\
\text { workers }\end{array}$ & China & $\begin{array}{l}\text { Establishment-b } \\
\text { ased } \\
\text { (restaurants, } \\
\text { barbershops, } \\
\text { massage } \\
\text { parlours etc.) }\end{array}$ & 454 FSWs & $\begin{array}{l}\text { Quantitative: } \\
\text { Self-administered } \\
\text { questionnaire }\end{array}$ & $\begin{array}{l}\text { Chi-squared tests, } \\
\text { Fisher exact tests, } \\
\text { analysis of } \\
\text { variance, } \\
\text { ordinal/binary } \\
\text { logistic regression }\end{array}$ & N/A \\
\hline $\begin{array}{l}\text { Yang, Y. et al. } \\
\text { (2016) }\end{array}$ & $\begin{array}{l}\text { Journal } \\
\text { Article }\end{array}$ & $\begin{array}{l}\text { Male } \\
\text { clients of } \\
\text { FSWs }\end{array}$ & China & N/A & $\begin{array}{l}584 \text { male clients } \\
\text { of FSWs }\end{array}$ & $\begin{array}{l}\text { Quantitative: } \\
\text { Structured } \\
\text { interviews }\end{array}$ & $\begin{array}{l}\text { Bivariate/Multivar } \\
\text { iate logistic } \\
\text { regression }\end{array}$ & Social Cognitive Theory \\
\hline Ye, X. et al. (2012) & $\begin{array}{l}\text { Journal } \\
\text { Article }\end{array}$ & $\begin{array}{l}\text { Female } \\
\text { sex } \\
\text { workers }\end{array}$ & China & $\begin{array}{l}\text { Small sex } \\
\text { establishments: } \\
\text { shampoo wash } \\
\text { rooms, massage } \\
\text { parlours, hair } \\
\text { salons }\end{array}$ & 504 FSWs & $\begin{array}{l}\text { Quantitative: } \\
\text { Survey and } \\
\text { interview }\end{array}$ & $\begin{array}{l}\text { Chi-squared tests, } \\
\text { multivariate } \\
\text { logistic regression }\end{array}$ & N/A \\
\hline $\begin{array}{l}\text { Zhang, H. et al. } \\
\text { (2011) }\end{array}$ & $\begin{array}{l}\text { Journal } \\
\text { Article }\end{array}$ & $\begin{array}{l}\text { Female } \\
\text { sex } \\
\text { workers }\end{array}$ & China & N/A & 427 FSWs & $\begin{array}{l}\text { Quantitative: } \\
\text { Self-administered } \\
\text { questionnaires }\end{array}$ & $\begin{array}{l}\text { Descriptive } \\
\text { statistics, } \\
\text { Confirmatory } \\
\text { factor analysis }\end{array}$ & $\begin{array}{l}\text { Information-Motivation-Beh } \\
\text { avioral Skills (IMB) model }\end{array}$ \\
\hline $\begin{array}{l}\text { Zhao, J. et al. } \\
\text { (2012) }\end{array}$ & $\begin{array}{l}\text { Journal } \\
\text { Article }\end{array}$ & $\begin{array}{l}\text { Female } \\
\text { sex } \\
\text { workers }\end{array}$ & China & $\begin{array}{l}\text { Low-tier } \\
\text { entertainment } \\
\text { Establishments }\end{array}$ & $\begin{array}{l}427 \quad \text { FSWS } \\
\text { recruited, } 363 \\
\text { valid } \\
\text { questionnaires }\end{array}$ & $\begin{array}{l}\text { Quantitative: } \\
\text { Self-administered } \\
\text { Questionnaires }\end{array}$ & $\begin{array}{l}\text { Descriptive } \\
\text { statistics, } \\
\text { Structural } \\
\text { equation model } \\
((\text { SEM)Confirmat } \\
\text { ory factor } \\
\text { analysis, } \\
\text { standardized } \\
\text { regression, }\end{array}$ & Health Belief Model \\
\hline
\end{tabular}




\begin{tabular}{|c|c|c|c|c|c|c|c|c|}
\hline & & & & & & & Cronbach's alpha) & \\
\hline $\begin{array}{l}\text { Zhao, R. et al. } \\
\text { (2008) }\end{array}$ & $\begin{array}{l}\text { Journal } \\
\text { Article }\end{array}$ & $\begin{array}{l}\text { Female } \\
\text { sex } \\
\text { workers }\end{array}$ & China & $\begin{array}{l}\text { Establishment } \\
\text { based } \\
\text { (restaurants, } \\
\text { barbershops, } \\
\text { shampoo salons, } \\
\text { massage } \\
\text { parlours) }\end{array}$ & 309 FSWs & $\begin{array}{l}\text { Quantitative: } \\
\text { Self-administered } \\
\text { questionnaire }\end{array}$ & $\begin{array}{l}\text { Chi-squared, } \\
\text { Fisher's exact } \\
\text { tests, binary } \\
\text { logistic regression }\end{array}$ & $\begin{array}{l}\text { Bandura's Theory of } \\
\text { Self-Efficacy }\end{array}$ \\
\hline $\begin{array}{l}\text { Zhou, C. et al. } \\
\text { (2019) }\end{array}$ & $\begin{array}{l}\text { Journal } \\
\text { Article }\end{array}$ & $\begin{array}{l}\text { Female } \\
\text { sex } \\
\text { workers }\end{array}$ & China & $\begin{array}{l}\text { Low-tier venues } \\
\text { (massage } \\
\text { parlours, hair } \\
\text { salons etc.) }\end{array}$ & $\begin{array}{l}312 \text { low-fee } \\
\text { FSWs }\end{array}$ & $\begin{array}{l}\text { Quantitative: } \\
\text { Questionnaire }\end{array}$ & $\begin{array}{l}\text { Descriptive } \\
\text { statistics, logistic } \\
\text { regression, } \\
\text { chi-squared tests }\end{array}$ & N/A \\
\hline
\end{tabular}

\section{Study Quality}

As shown in Table 3 the QAT included six major assessment criteria: a) selection bias, b) study design, c) confounders, d) blinding, e) data collection methods and f) withdrawals and drop-outs. Fifteen specific quality assessment sub-criteria were weighted according to the guidelines described for use of the QAT (Thomas, 2003). Global scores for each major criterion, based on the responses to the sub-criteria, provided an overall indication of a study's quality level: Strong = 1, Moderate $=2$ and Weak $=3$. Although study quality varied significantly across the 15 quality assessment sub-criteria, all 40 studies assessed were found to be weak given that they attracted more than two weak scores across the six major criteria indicated above.

For qualitative studies, the Mixed Methods Appraisal Tool (MMAT), as shown in Table 4, was used to appraise the quality and reliability of the studies included (Hong et al., 2018). Two screening questions assess the feasibility of using the MMAT for appraisal, followed by five questions specific to qualitative studies. Responses are categorized into three groups; Yes, No and Can’t Tell.

Table 3. Quality Assessment Results

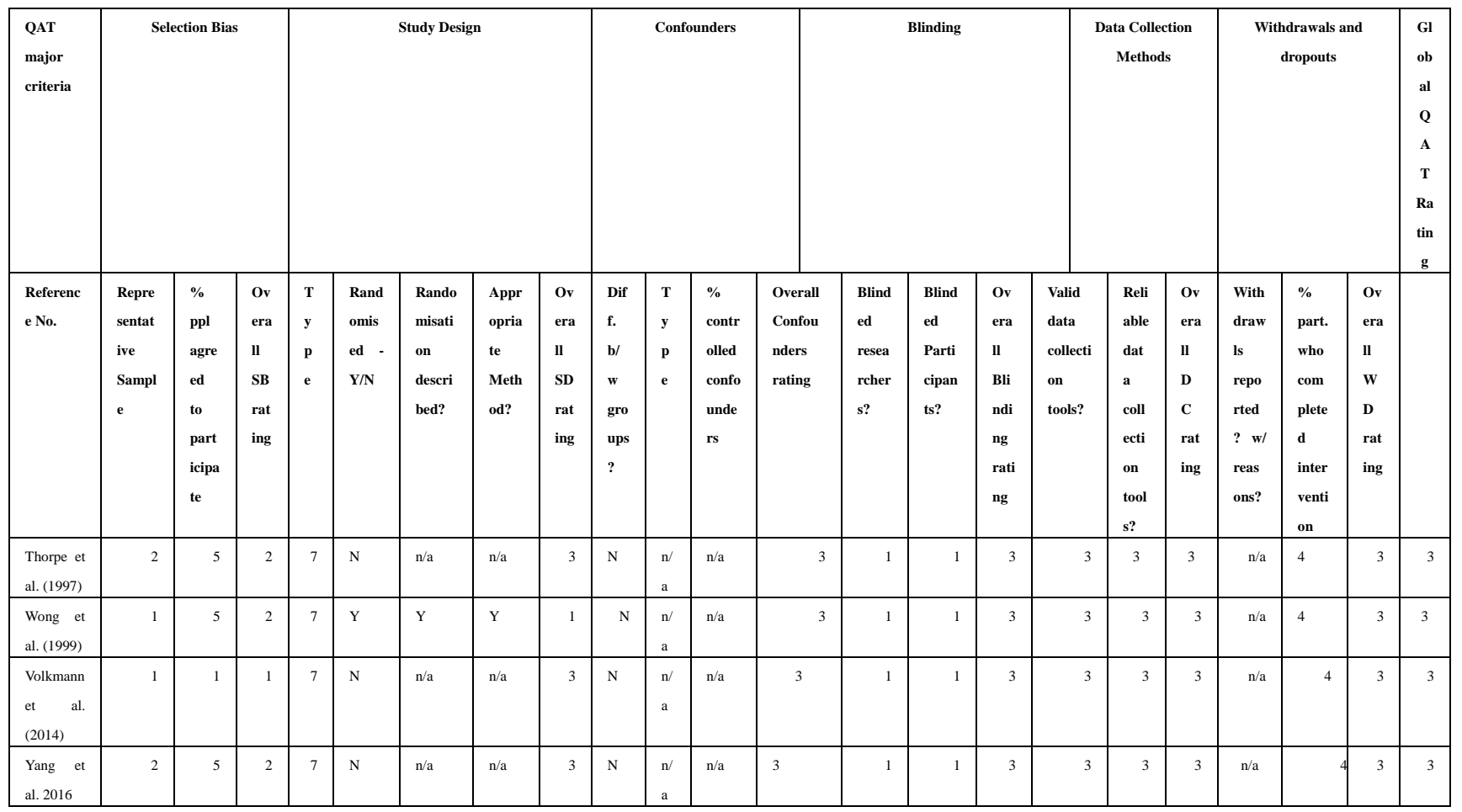




\begin{tabular}{|c|c|c|c|c|c|c|c|c|c|c|c|c|c|c|c|c|c|c|c|c|c|c|}
\hline $\begin{array}{l}\text { Wee et al. } \\
(2004)\end{array}$ & 1 & 1 & 1 & 7 & $\mathrm{~N}$ & $\mathrm{n} / \mathrm{a}$ & $\mathrm{n} / \mathrm{a}$ & 3 & $\mathrm{~N}$ & $\begin{array}{l}\mathrm{n} / \\
\mathrm{a}\end{array}$ & $\mathrm{n} / \mathrm{a}$ & 3 & 1 & 1 & 3 & 3 & 3 & 3 & $\mathrm{n} / \mathrm{a}$ & 4 & 3 & 3 \\
\hline $\begin{array}{l}\text { Qu et al. } \\
\text { (2002) }\end{array}$ & 1 & 1 & 1 & 7 & $\mathrm{~N}$ & $\mathrm{n} / \mathrm{a}$ & $\mathrm{n} / \mathrm{a}$ & 3 & $\mathrm{~N}$ & $\begin{array}{l}\mathrm{n} / \\
\mathrm{a}\end{array}$ & $\mathrm{n} / \mathrm{a}$ & 3 & 1 & 1 & 3 & 3 & 3 & 3 & $\mathrm{n} / \mathrm{a}$ & 4 & 3 & 3 \\
\hline $\begin{array}{l}\text { Zhao et } \\
\text { al. (2012) }\end{array}$ & 1 & 5 & 2 & 7 & $\mathrm{~N}$ & $\mathrm{n} / \mathrm{a}$ & $\mathrm{n} / \mathrm{a}$ & 3 & $\mathrm{~N}$ & $\begin{array}{l}\mathrm{n} / \\
\mathrm{a}\end{array}$ & $\mathrm{n} / \mathrm{a}$ & 3 & 1 & 1 & 3 & 3 & 3 & 3 & $\mathrm{n} / \mathrm{a}$ & 4 & 3 & 3 \\
\hline $\begin{array}{l}\text { Barringto } \\
\mathrm{n} \text { et al. } \\
(2009)\end{array}$ & 2 & 5 & 2 & 7 & $\mathrm{~N}$ & $\mathrm{n} / \mathrm{a}$ & $\mathrm{n} / \mathrm{a}$ & 3 & $\mathrm{~N}$ & $\begin{array}{l}\mathrm{n} / \\
\mathrm{a}\end{array}$ & $\mathrm{n} / \mathrm{a}$ & 3 & 1 & 1 & 3 & 3 & 3 & 3 & $\mathrm{n} / \mathrm{a}$ & 4 & 3 & 3 \\
\hline $\begin{array}{l}\text { Grosso et } \\
\text { al. (2015) }\end{array}$ & 2 & 5 & 2 & 7 & $\mathrm{~N}$ & n/a & $\mathrm{n} / \mathrm{a}$ & 3 & $\mathrm{~N}$ & $\begin{array}{l}\mathrm{n} / \\
\mathrm{a}\end{array}$ & n/a & 3 & 1 & 1 & 3 & 3 & 3 & 3 & $\mathrm{n} / \mathrm{a}$ & 4 & 3 & 3 \\
\hline $\begin{array}{l}\text { Rwema et } \\
\text { al. (2019) }\end{array}$ & 2 & 5 & 2 & 7 & $\mathrm{~N}$ & $\mathrm{n} / \mathrm{a}$ & $\mathrm{n} / \mathrm{a}$ & 3 & $\mathrm{~N}$ & $\begin{array}{l}\mathrm{n} / \\
\mathrm{a}\end{array}$ & $\mathrm{n} / \mathrm{a}$ & 3 & 1 & 1 & 3 & 3 & 3 & 3 & $\mathrm{n} / \mathrm{a}$ & 4 & 3 & 3 \\
\hline $\begin{array}{l}\text { Zhang et } \\
\text { al. (2011) }\end{array}$ & 2 & 1 & 2 & 7 & $\mathrm{~N}$ & $\mathrm{n} / \mathrm{a}$ & $\mathrm{n} / \mathrm{a}$ & 3 & $\mathrm{~N}$ & $\begin{array}{l}\mathrm{n} / \\
\mathrm{a}\end{array}$ & $\mathrm{n} / \mathrm{a}$ & 3 & 1 & 1 & 3 & 3 & 3 & 3 & $\mathrm{n} / \mathrm{a}$ & 4 & 3 & 3 \\
\hline $\begin{array}{l}\text { Zhao et } \\
\text { al. (2008) }\end{array}$ & 2 & 2 & 3 & 7 & $\mathrm{~N}$ & $\mathrm{n} / \mathrm{a}$ & $\mathrm{n} / \mathrm{a}$ & 3 & $\mathrm{~N}$ & $\begin{array}{l}\mathrm{n} / \\
\mathrm{a}\end{array}$ & $\mathrm{n} / \mathrm{a}$ & 3 & 1 & 1 & 3 & 3 & 3 & 3 & $\mathrm{n} / \mathrm{a}$ & 4 & 3 & 3 \\
\hline $\begin{array}{l}\text { Bharat et } \\
\text { al. (2013) }\end{array}$ & 1 & 1 & 1 & 7 & $\mathrm{~N}$ & n/a & $\mathrm{n} / \mathrm{a}$ & 3 & $\mathrm{~N}$ & $\begin{array}{l}\mathrm{n} / \\
\mathrm{a}\end{array}$ & $\mathrm{n} / \mathrm{a}$ & 3 & 1 & 1 & 3 & 3 & 3 & 3 & $\mathrm{n} / \mathrm{a}$ & 4 & 3 & 3 \\
\hline $\begin{array}{l}\text { Cheng et } \\
\text { al. (2010) }\end{array}$ & 2 & 2 & 3 & 7 & $\mathrm{~N}$ & $\mathrm{n} / \mathrm{a}$ & $\mathrm{n} / \mathrm{a}$ & 3 & $\mathrm{~N}$ & $\begin{array}{l}\mathrm{n} / \\
\mathrm{a}\end{array}$ & $\mathrm{n} / \mathrm{a}$ & 3 & 1 & 1 & 3 & 3 & 3 & 3 & $\mathrm{n} / \mathrm{a}$ & 4 & 3 & 3 \\
\hline $\begin{array}{l}\text { Shannon } \\
\text { et al. } \\
(2009)\end{array}$ & 2 & 1 & 2 & 7 & $\mathrm{~N}$ & $\mathrm{n} / \mathrm{a}$ & $\mathrm{n} / \mathrm{a}$ & 3 & $\mathrm{~N}$ & $\begin{array}{l}\mathrm{n} / \\
\mathrm{a}\end{array}$ & $\mathrm{n} / \mathrm{a}$ & 3 & 1 & 1 & 3 & 3 & 3 & 3 & $\mathrm{n} / \mathrm{a}$ & 4 & 3 & 3 \\
\hline $\begin{array}{l}\text { Grayman } \\
\text { et al. } \\
(2005)\end{array}$ & 2 & 5 & 3 & 7 & $\mathrm{~N}$ & $\mathrm{n} / \mathrm{a}$ & $\mathrm{n} / \mathrm{a}$ & 3 & $\mathrm{~N}$ & $\begin{array}{l}\mathrm{n} / \\
\mathrm{a}\end{array}$ & $\mathrm{n} / \mathrm{a}$ & 3 & 1 & 1 & 3 & 3 & 3 & 3 & $\mathrm{n} / \mathrm{a}$ & 4 & 3 & 3 \\
\hline $\begin{array}{l}\text { Ye et al. } \\
(2012)\end{array}$ & 2 & 5 & 3 & 7 & $\mathrm{~N}$ & $\mathrm{n} / \mathrm{a}$ & $\mathrm{n} / \mathrm{a}$ & 3 & $\mathrm{~N}$ & $\begin{array}{l}\mathrm{n} / \\
\mathrm{a}\end{array}$ & $\mathrm{n} / \mathrm{a}$ & 3 & 1 & 1 & 3 & 3 & 3 & 3 & $\mathrm{n} / \mathrm{a}$ & 4 & 3 & 3 \\
\hline $\begin{array}{l}\text { Decker et } \\
\text { al. (2019) }\end{array}$ & 2 & 5 & 3 & 7 & $\mathrm{~N}$ & $\mathrm{n} / \mathrm{n}$ & $\mathrm{n} / \mathrm{a}$ & 3 & $\mathrm{~N}$ & $\begin{array}{l}\mathrm{n} / \\
\mathrm{a}\end{array}$ & $\mathrm{n} / \mathrm{a}$ & 3 & 1 & 1 & 3 & 3 & 3 & 3 & n/a & 4 & 3 & 3 \\
\hline $\begin{array}{l}\text { Parcesepe } \\
\text { et al. } \\
(2016)\end{array}$ & 2 & 5 & 2 & 7 & $\mathrm{~N}$ & $\mathrm{n} / \mathrm{a}$ & $\mathrm{n} / \mathrm{a}$ & 3 & $\mathrm{~N}$ & $\begin{array}{l}\mathrm{n} / \\
\mathrm{a}\end{array}$ & $\mathrm{n} / \mathrm{a}$ & 3 & 1 & 1 & 3 & 3 & 3 & 3 & $\mathrm{n} / \mathrm{a}$ & 4 & 3 & 3 \\
\hline $\begin{array}{l}\text { Bruce et } \\
\text { al. (2011) }\end{array}$ & 2 & 5 & 3 & 7 & $\mathrm{~N}$ & $\mathrm{n} / \mathrm{a}$ & $\mathrm{n} / \mathrm{a}$ & 3 & $\mathrm{~N}$ & $\begin{array}{l}\mathrm{n} / \\
\mathrm{a}\end{array}$ & $\mathrm{n} / \mathrm{a}$ & 3 & 1 & 1 & 3 & 3 & 3 & 3 & $\mathrm{n} / \mathrm{a}$ & 4 & 3 & 3 \\
\hline $\begin{array}{l}\text { Jung } \\
\text { (2013) }\end{array}$ & 2 & 5 & 2 & 7 & $\mathrm{~N}$ & $\mathrm{n} / \mathrm{a}$ & $\mathrm{n} / \mathrm{a}$ & 3 & $\mathrm{~N}$ & $\begin{array}{l}\mathrm{n} / \\
\mathrm{a}\end{array}$ & $\mathrm{n} / \mathrm{a}$ & 3 & 1 & 1 & 3 & 3 & 3 & 3 & $\mathrm{n} / \mathrm{a}$ & 4 & 3 & 3 \\
\hline $\begin{array}{l}\text { Wang et } \\
\text { al. (2009) }\end{array}$ & 2 & 2 & 2 & 7 & $\mathrm{~N}$ & $\mathrm{n} / \mathrm{a}$ & $\mathrm{n} / \mathrm{a}$ & 3 & $\mathrm{~N}$ & $\begin{array}{l}\mathrm{n} / \\
\mathrm{a}\end{array}$ & $\mathrm{n} / \mathrm{a}$ & 3 & 1 & 1 & 3 & 3 & 3 & 3 & $\mathrm{n} / \mathrm{a}$ & 4 & 3 & 3 \\
\hline $\begin{array}{l}\text { Yang et } \\
\text { al. (2005) }\end{array}$ & 2 & 2 & 2 & 7 & $\mathrm{~N}$ & n/a & $\mathrm{n} / \mathrm{a}$ & 3 & $\mathrm{~N}$ & $\begin{array}{l}\mathrm{n} / \\
\mathrm{a}\end{array}$ & $\mathrm{n} / \mathrm{a}$ & 3 & 1 & 1 & 3 & 3 & 3 & 3 & $\mathrm{n} / \mathrm{a}$ & 4 & 3 & 3 \\
\hline $\begin{array}{l}\text { Munoz et } \\
\text { al. (2010) }\end{array}$ & 1 & 5 & 2 & 1 & $\mathrm{Y}$ & $\mathrm{N}$ & $\mathrm{N}$ & 2 & $\mathrm{~N}$ & $\begin{array}{l}\mathrm{n} / \\
\mathrm{a}\end{array}$ & $\mathrm{n} / \mathrm{a}$ & 3 & 1 & 2 & 2 & 1 & 3 & 2 & $\mathrm{n} / \mathrm{a}$ & 4 & 3 & 3 \\
\hline $\begin{array}{l}\text { Vejella et } \\
\text { al. (2015) }\end{array}$ & 1 & 5 & 2 & 7 & $\mathrm{~N}$ & $\mathrm{n} / \mathrm{a}$ & $\mathrm{n} / \mathrm{a}$ & 3 & $\mathrm{~N}$ & $\begin{array}{l}\mathrm{n} / \\
\mathrm{a}\end{array}$ & $\mathrm{n} / \mathrm{a}$ & 3 & 1 & 1 & 3 & 3 & 3 & 3 & $\mathrm{n} / \mathrm{a}$ & 4 & 3 & 3 \\
\hline $\begin{array}{l}\text { Saggurti } \\
\text { et al. } \\
(2013)\end{array}$ & 1 & 1 & 1 & 7 & $\mathrm{~N}$ & $\mathrm{n} / \mathrm{a}$ & $\mathrm{n} / \mathrm{a}$ & 3 & $\mathrm{~N}$ & $\begin{array}{l}\mathrm{n} / \\
\mathrm{a}\end{array}$ & $\mathrm{n} / \mathrm{a}$ & 3 & 1 & 1 & 3 & 3 & 3 & 3 & $\mathrm{n} / \mathrm{a}$ & 4 & 3 & 3 \\
\hline $\begin{array}{l}\text { Urada et } \\
\text { al. (2012) }\end{array}$ & 1 & 1 & 1 & 7 & $\mathrm{~N}$ & $\mathrm{n} / \mathrm{a}$ & $\mathrm{n} / \mathrm{a}$ & 3 & $\mathrm{~N}$ & $\begin{array}{l}\mathrm{n} / \\
\mathrm{a}\end{array}$ & $\mathrm{n} / \mathrm{a}$ & 3 & 1 & 1 & 3 & 3 & 3 & 3 & $\mathrm{n} / \mathrm{a}$ & 4 & 3 & 3 \\
\hline $\begin{array}{l}\text { Bui et al. } \\
\text { (2012) }\end{array}$ & 2 & 1 & 2 & 7 & $\mathrm{~N}$ & $\mathrm{n} / \mathrm{a}$ & $\mathrm{n} / \mathrm{a}$ & 3 & $\mathrm{~N}$ & $\begin{array}{l}\mathrm{n} / \\
\mathrm{a}\end{array}$ & $\mathrm{n} / \mathrm{a}$ & 3 & 1 & 1 & 3 & 3 & 3 & 3 & $\mathrm{n} / \mathrm{a}$ & 4 & 3 & 3 \\
\hline $\begin{array}{l}\text { Darbinya } \\
\mathrm{n} \quad \text { et al. } \\
(2011)\end{array}$ & 2 & 5 & 2 & 7 & $\mathrm{~N}$ & $\mathrm{n} / \mathrm{a}$ & $\mathrm{n} / \mathrm{a}$ & 3 & $\mathrm{~N}$ & $\begin{array}{l}\mathrm{n} / \\
\mathrm{a}\end{array}$ & $\mathrm{n} / \mathrm{a}$ & 3 & 1 & 1 & 3 & 1 & 3 & 2 & $\mathrm{n} / \mathrm{a}$ & 4 & 3 & 3 \\
\hline $\begin{array}{l}\text { Alam et } \\
\text { al. (2013) }\end{array}$ & 1 & 1 & 1 & 7 & $\mathrm{~N}$ & $\mathrm{n} / \mathrm{a}$ & $\mathrm{n} / \mathrm{a}$ & 3 & $\mathrm{~N}$ & $\begin{array}{l}\mathrm{n} / \\
\mathrm{a}\end{array}$ & $\mathrm{n} / \mathrm{a}$ & 3 & 1 & 1 & 3 & 1 & 3 & 2 & $\mathrm{n} / \mathrm{a}$ & 4 & 3 & 3 \\
\hline $\begin{array}{l}\text { Ford et a.l } \\
(2000)\end{array}$ & 2 & 1 & 1 & 7 & $\mathrm{~N}$ & $\mathrm{n} / \mathrm{a}$ & $\mathrm{n} / \mathrm{a}$ & 3 & $\mathrm{~N}$ & $\begin{array}{l}\mathrm{n} / \\
\mathrm{a}\end{array}$ & $\mathrm{n} / \mathrm{a}$ & 3 & 1 & 1 & 3 & 1 & 3 & 2 & $\mathrm{n} / \mathrm{a}$ & 4 & 3 & 3 \\
\hline $\begin{array}{l}\text { Sedyanin } \\
\text { gsih-Mah } \\
\text { amit et al. } \\
\text { (1999) }\end{array}$ & 2 & 2 & 2 & 7 & $\mathrm{Y}$ & $\mathrm{Y}$ & $\mathrm{Y}$ & 1 & $\mathrm{~N}$ & $\begin{array}{l}\mathrm{n} / \\
\mathrm{a}\end{array}$ & $\mathrm{n} / \mathrm{a}$ & 3 & 1 & 1 & 3 & 1 & 3 & 2 & $\mathrm{n} / \mathrm{a}$ & 4 & 3 & 3 \\
\hline
\end{tabular}




\begin{tabular}{|c|c|c|c|c|c|c|c|c|c|c|c|c|c|c|c|c|c|c|c|c|c|c|}
\hline $\begin{array}{l}\text { Zhou et } \\
\text { al. (2019) }\end{array}$ & 2 & 5 & 2 & 7 & $\mathrm{~N}$ & $\mathrm{n} / \mathrm{a}$ & $\mathrm{n} / \mathrm{a}$ & 3 & $\mathrm{~N}$ & $\mathrm{n} /$ & $\mathrm{n} / \mathrm{a}$ & 3 & 1 & 1 & 3 & 1 & 3 & 2 & n/a & 4 & 3 & 3 \\
\hline $\begin{array}{l}\text { Blankens } \\
\text { hip et al. } \\
(2008) \\
\end{array}$ & 1 & 5 & 2 & 7 & $\mathrm{~N}$ & $\mathrm{n} / \mathrm{a}$ & $\mathrm{n} / \mathrm{a}$ & 3 & $\mathrm{~N}$ & $\begin{array}{l}\mathrm{n} / \\
\mathrm{a}\end{array}$ & $\mathrm{n} / \mathrm{a}$ & 3 & 1 & 1 & 3 & 1 & 3 & 2 & n/a & 4 & 3 & 3 \\
\hline $\begin{array}{l}\text { Elmes et } \\
\text { al. (2014) }\end{array}$ & 2 & 5 & 2 & 7 & $\mathrm{~N}$ & $\mathrm{n} / \mathrm{a}$ & $\mathrm{n} / \mathrm{a}$ & 3 & $\mathrm{~N}$ & $\mathrm{n} /$ & $\mathrm{n} / \mathrm{a}$ & 3 & 1 & 1 & 3 & 1 & 3 & 2 & n/a & 4 & 3 & 3 \\
\hline $\begin{array}{l}\text { Kamal et } \\
\text { al. (2015) }\end{array}$ & 1 & 1 & 1 & 7 & $\mathrm{~N}$ & $\mathrm{n} / \mathrm{a}$ & $\mathrm{n} / \mathrm{a}$ & 3 & $\mathrm{~N}$ & $\mathrm{n} /$ & $\mathrm{n} / \mathrm{a}$ & 3 & 1 & 1 & 3 & 1 & 3 & 2 & n/a & 4 & 3 & 3 \\
\hline $\begin{array}{l}\text { Kerrigan } \\
\text { et al. } \\
(2003)\end{array}$ & 1 & 5 & 2 & 7 & $\mathrm{~N}$ & $\mathrm{n} / \mathrm{a}$ & $\mathrm{n} / \mathrm{a}$ & 3 & $\mathrm{~N}$ & $\begin{array}{l}\mathrm{n} / \\
\mathrm{a}\end{array}$ & $\mathrm{n} / \mathrm{a}$ & 3 & 1 & 1 & 3 & 1 & 3 & 2 & n/a & 4 & 3 & 3 \\
\hline $\begin{array}{l}\text { Lausevic } \\
\text { et al. } \\
(2016)\end{array}$ & 2 & 5 & 2 & 7 & $\mathrm{~N}$ & $\mathrm{n} / \mathrm{a}$ & $\mathrm{n} / \mathrm{a}$ & 3 & $\mathrm{~N}$ & $\begin{array}{l}\mathrm{n} / \\
\mathrm{a}\end{array}$ & $\mathrm{n} / \mathrm{a}$ & 3 & 1 & 1 & 3 & 1 & 3 & 2 & n/a & 4 & 3 & 3 \\
\hline $\begin{array}{l}\text { Lee et al. } \\
(2010)\end{array}$ & 2 & 1 & 2 & 7 & $\mathrm{~N}$ & $\mathrm{n} / \mathrm{a}$ & $\mathrm{n} / \mathrm{a}$ & 3 & $\mathrm{~N}$ & $\begin{array}{l}\mathrm{n} / \\
\mathrm{a}\end{array}$ & $\mathrm{n} / \mathrm{a}$ & 3 & 1 & 1 & 3 & 1 & 3 & 2 & n/a & 4 & 3 & 3 \\
\hline $\begin{array}{l}\text { Busza et } \\
\text { al. (2010) }\end{array}$ & 2 & 5 & 3 & 7 & $\mathrm{~N}$ & $\mathrm{n} / \mathrm{a}$ & $\mathrm{n} / \mathrm{a}$ & 3 & $\mathrm{~N}$ & $\mathrm{n} /$ & $\mathrm{n} / \mathrm{a}$ & 3 & 1 & 1 & 3 & 3 & 3 & 3 & n/a & 4 & 3 & 3 \\
\hline
\end{tabular}

Table 4. The Mixed Methods Appraisal Tool (MMAT) Quality Assessment for Included Studies

\begin{tabular}{|c|c|c|c|c|c|}
\hline Study & Study Design & Methodological Quality Criteria & $\begin{array}{l}\text { Yes (low risk of } \\
\text { bias) }\end{array}$ & No (high risk of bias) & Unclear \\
\hline \multirow[t]{5}{*}{$\begin{array}{l}\text { Bailey et al. } \\
\text { (2018) }\end{array}$} & \multirow[t]{5}{*}{ Qualitative } & $\begin{array}{l}\text { Appropriate approach to answer research } \\
\text { question }\end{array}$ & $\mathbf{X}$ & & \\
\hline & & Adequate data collection methods & $\mathbf{X}$ & & \\
\hline & & Findings are adequately derived from data & $\mathbf{X}$ & & \\
\hline & & Interpretations of results substantiated by data & $\mathbf{X}$ & & \\
\hline & & $\begin{array}{l}\text { Coherence between qualitative data sources, } \\
\text { collection, analysis and interpretation }\end{array}$ & & & $\mathbf{X}$ \\
\hline \multirow[t]{5}{*}{$\begin{array}{l}\text { Choi et al. } \\
(2007)\end{array}$} & \multirow[t]{5}{*}{ Qualitative } & $\begin{array}{l}\text { Appropriate approach to answer research } \\
\text { question }\end{array}$ & $\mathbf{X}$ & & \\
\hline & & Adequate data collection methods & $\mathbf{X}$ & & \\
\hline & & Findings are adequately derived from data & $\mathbf{X}$ & & \\
\hline & & Interpretations of results substantiated by data & $\mathbf{X}$ & & \\
\hline & & $\begin{array}{l}\text { Coherence between qualitative data sources, } \\
\text { collection, analysis and interpretation }\end{array}$ & $\mathbf{X}$ & & \\
\hline \multirow[t]{5}{*}{$\begin{array}{l}\text { Cusick et al. } \\
\text { (1998) }\end{array}$} & \multirow[t]{5}{*}{ Qualitative } & $\begin{array}{l}\text { Appropriate approach to answer research } \\
\text { question }\end{array}$ & $\mathbf{X}$ & & \\
\hline & & Adequate data collection methods & $\mathbf{X}$ & & \\
\hline & & Findings are adequately derived from data & $\mathbf{X}$ & & \\
\hline & & Interpretations of results substantiated by data & $\mathbf{X}$ & & \\
\hline & & $\begin{array}{l}\text { Coherence between qualitative data sources, } \\
\text { collection, analysis and interpretation }\end{array}$ & $\mathbf{X}$ & & \\
\hline \multirow[t]{4}{*}{$\begin{array}{l}\text { Fauk et al. } \\
(2018)\end{array}$} & \multirow[t]{4}{*}{ Qualitative } & $\begin{array}{l}\text { Appropriate approach to answer research } \\
\text { question }\end{array}$ & $\mathbf{X}$ & & \\
\hline & & Adequate data collection methods & $\mathbf{X}$ & & \\
\hline & & Findings are adequately derived from data & $\mathbf{X}$ & & \\
\hline & & Interpretations of results substantiated by data & $\mathbf{X}$ & & \\
\hline
\end{tabular}




\begin{tabular}{|c|c|c|c|c|}
\hline & & $\begin{array}{l}\text { Coherence between qualitative data sources, } \\
\text { collection, analysis and interpretation }\end{array}$ & $\mathbf{X}$ & \\
\hline \multirow[t]{5}{*}{$\begin{array}{l}\text { Ghimire et al. } \\
\text { (2011) }\end{array}$} & \multirow[t]{5}{*}{ Qualitative } & $\begin{array}{l}\text { Appropriate approach to answer research } \\
\text { question }\end{array}$ & $\mathbf{X}$ & \\
\hline & & Adequate data collection methods & & $\mathbf{x}$ \\
\hline & & Findings are adequately derived from data & $\mathbf{X}$ & \\
\hline & & Interpretations of results substantiated by data & $\mathbf{x}$ & \\
\hline & & $\begin{array}{l}\text { Coherence between qualitative data sources, } \\
\text { collection, analysis and interpretation }\end{array}$ & $\mathbf{x}$ & \\
\hline \multirow[t]{5}{*}{$\begin{array}{l}\text { Maher et al. } \\
\text { (2013) }\end{array}$} & \multirow[t]{5}{*}{ Qualitative } & $\begin{array}{l}\text { Appropriate approach to answer research } \\
\text { question }\end{array}$ & $\mathbf{x}$ & \\
\hline & & Adequate data collection methods & $\mathbf{x}$ & \\
\hline & & Findings are adequately derived from data & $\mathbf{x}$ & \\
\hline & & Interpretations of results substantiated by data & $\mathbf{x}$ & \\
\hline & & $\begin{array}{l}\text { Coherence between qualitative data sources, } \\
\text { collection, analysis and interpretation }\end{array}$ & $\mathbf{x}$ & \\
\hline \multirow[t]{5}{*}{$\begin{array}{l}\text { Mathenjwa et } \\
\text { al. (2012) }\end{array}$} & \multirow[t]{5}{*}{ Qualitative } & $\begin{array}{l}\text { Appropriate approach to answer research } \\
\text { question }\end{array}$ & $\mathbf{x}$ & \\
\hline & & Adequate data collection methods & $\mathbf{X}$ & \\
\hline & & Findings are adequately derived from data & $\mathbf{x}$ & \\
\hline & & Interpretations of results substantiated by data & $\mathbf{X}$ & \\
\hline & & $\begin{array}{l}\text { Coherence between qualitative data sources, } \\
\text { collection, analysis and interpretation }\end{array}$ & $\mathbf{x}$ & \\
\hline
\end{tabular}

\section{Major findings}

There were ten major themes identified following data extraction of the included studies. These were perceived self-efficacy, type of relationship between the FSW and client, condom use and attitudes, violence and abuse, the location of sexual encounters, support from peers and managers, knowledge and perceived risk, male clients, drug and alcohol use, criminalization and price and payment.

\section{Theme 1: Perceived self-efficacy}

Perceived self-efficacy was one of the most prominent themes that appeared across a number of studies. Various factors were identified as barriers or promoters of self-efficacy including agency, negotiation skills and peer solidarity and collective agency/efficacy.

\section{Agency}

Cheng and Mak (2010) found that FSWs with higher condom use self-efficacy were 24 times less likely to use condoms inconsistently (OR 0.041, 95\% CI: 0.006-0.288, p< 0.001). While there are many accounts where FSWs find it difficult to negotiate with or refuse clients for various reasons, there are also many women who show agency and confidence in choosing clients as seen in the extract below,

"I am an attractive lady. I have passed my school leaving certificate (Grade 10). I work in a dance restaurant and I know many good looking and high-class men. I charge more than Rs. 2000 per client. I use condoms every time. If a client refuses to use a condom, I get another client". (Ghimire, Smith, van Teijlingen, Dahal, \& Luitel, 2011, p.3)

In a study conducted in Nepal in 2011, the main reasons for not using condoms was reported to be either for financial reasons or low-self efficacy to negotiate condom use with clients (Ghimire et al. 2011).

\section{Negotiation Skills}


FSWs who perceived pressure by managers or clients reported lower self-efficacy related to condom use with clients (Cheng \& Mak 2010). Negotiation skills by FSWs were one of the most common factors contributing to condom use in a commercial setting. The resistance of clients towards condom use was shown to negatively affect negotiation skills of the FSW (Cheng \& Mak, 2010). Zhao, Wang, Fang, Li \& Stanton (2008) found that 52\% of FSWs in their study would refuse to have sex with a client who was not willing to wear condoms, while $62 \%$ reported that they were able to persuade a client who had initially refused to use a condom. In this study $39 \%$ of FSWs cited clients' refusal as the main reason that condoms were not used (Zhao et al. 2008). Participants in a 2017 study based in Jamaica stated that condom use was a standard negotiation procedure; second to agreeing on the price (Bailey \& Figueroa, 2018). Negotiation skills were deemed an essential part of the job, as almost all participants could describe requests by clients for unprotected sex (Bailey \& Figueroa, 2018).

Various negotiation strategies were discussed with the main three being fear arousal, sweet talk and no condom equals no sex (Maher et al., 2013). Fear arousal involved appealing to the clients concern for his own health and his sense of responsibility to his family (Maher et al., 2013). In this strategy the sex worker would frame herself as a threat to the clients' health and family (Maher et al., 2013). Sweet talk involved consoling the client and 'with sweet words' and explaining to the client that they do not know or trust each other (Maher et al., 2013). The third strategy was straight refusal although this strategy may be more difficult for less experienced FSWs (Maher et al., 2013).

Peer solidarity and collective agency

Peer solidarity among FSWs was a common theme and found to be an important factor in consistent condom use (Bailey \& Figueroa 2018). FSWs who reported high levels of collective efficacy among peers were more likely to consistently use condoms (Saggurti et al., 2013). FSWs who reported high levels of collective efficacy showed an increase in consistent condom use when compared with peers who reported low levels of collective efficacy (Vejella, Patel, Saggurti \& Prabhakar, 2016). Peer solidarity was also shown to increase female condom use among FSWs with good social support networks that were open to discussing protection from STIs and other shared experiences (Busza \& Baker, 2004).

\section{Theme 2: Relationship Types}

The type of relationship between FSW and client had a significant impact on condom use. The major themes extracted from the type of relationship were relationship fluidity, the symbolism of condoms in varying relationship dynamics and special regular or "Sweetheart" relationships, as they are known in Cambodia.

\section{Relationship fluidity}

The distinction between a commercial partner and a special or regular customer was often difficult to define. Bailey \& Figueroa (2018) describes that the demarcations between client, regular client and main partner were fluid, noting that a transition from client to partner was evident in multiple discussions with FSWs. Some discussions found that regular clients had become as important or even more supportive than the main or steady partner of the FSW (Bailey \& Figueroa, 2018). The movement from client to a special regular client or partner is often accompanied by ceasing the use of condoms.

\section{Symbolism of condoms}

The symbolism of condoms was a prominent factor in many of the included studies. Condoms were described as giving a commercial nature to the relationship and are often how FSWs are able to differentiate between commercial partners and main or steady partners (Zhao et al. 2008; Thorpe, Ford, Fajans \& Wirawan, 1997). Many of the women included in these studies acknowledged condom use as a practical way to prevent unwanted pregnancy and disease transmission, however, their husbands or main partners associated condom use with a lack of trust and emotional connection and not allowing the relationship to be properly marital in the sense that they are preventing conception and producing children (Maher et al., 2013). Many of the FSWs agreed with this belief, with one woman describing the absence of a condom being symbolic of love. Although the risk of forgoing condom use was understood, as she was unsure about whether she was the sole sexual partner of her husband, not using a condom was a symbol of intimacy and love in this relationship (Maher et al., 2013). This point of view was a common theme with husbands or main partner of FSWs, with many partners believing that the use of a condom was symbolic of a lack of care or emotional connection (Maher et al., 2013). Due to strict family planning policies in China, condoms have primarily been used as a birth control method within the context of marriage, and when the distinction between love and commercial transaction is unclear, discontinuing the use of condoms is seen as symbolic of the transition from "sex as an exchange, to sex as an expression of love" (Choi \& Holroyd, 2007, p.499).

The use of condoms is also symbolic of trust, or a lack of, with one FSW describing the difficulty of discussing condom use with a long-term partner, stating that this discussion often brings about a question of trust and rather uses the threat of unwanted pregnancy to negotiate condom use (Mathenjwa \& Maharaj, 2012). In long-term relationships, condoms 
were described as being associated with negative connotations, being seen as a violation of trust and often creating suspicion when used in steady relationships (Mathenjwa \& Maharaj, 2012), with one-third (35\%) of FSWs in a study citing trust and emotional factors as the main reason for sex without condoms in steady or long-term relationships (Bruce et al., 2011).

\section{Special regular customer/Sweetheart relationships}

Sweetheart relationships have been described by some of the included studies, and the term is generally used to define a commercial relationship that has transitioned into a relationship with some form of emotional connection or attachment. In the 2010 Cambodian Behavioural Sentinel Surveillance, 48.3\% of FSWs had a sweetheart in the past year (Bui et al., 2013). Having some form of intimate or emotional relationship with a FSW has been found to be a prominent risk factor for a lack of consistent condom use (Yang,Yang, Latkin, Luan \& Nelson 2016). Barrington et al., (2009) found a significant negative association between having been in a relationship lasting more than 3 months with a female sex worker and consistent condom use (OR $0.45,95 \% \mathrm{CI}=0.25-0.81$ ). It is common for relationships of this nature to not always involve a monetary exchange for services, with special regular customers sometimes supporting FSWs in other ways. One FSW cites how a regular client has helped her:

"I have a few regular clients who I have an attachment for. They love me and come to me with me they don't want sex using condoms. I never press them as I am a widow. They have helped me in many ways and in raising my kids. So I cannot insist them using a condom". (Ghimire et al. 2011, p.4)

Theme 3: Condom use and attitudes

Condom attitudes, both positive and negative were represented in these studies. Belief in the efficacy of condoms, negative attitudes towards condom use and the use of the female condom were prominent themes the appeared in relation to condom use in the commercial setting.

\section{Belief in the efficacy of condoms}

Some FSWs expressed positive attitudes towards condoms and enabled them to feel safe and protected (Bailey \& Figueroa, 2018). Belief in the efficacy of condoms was a strong predictor for consistent condom use, however, despite high awareness and knowledge of STI's, condoms were still often abandoned for various reasons by both sex workers and clients (Bailey \& Figueroa, 2018). Some participants did not trust male condoms as they had experienced breakage and did not allow them to enjoy the sexual experience (Mathenjwa \& Maharaj, 2012).

\section{Negative attitudes towards condoms}

While some participants relied on condoms for contraceptive purposes (Mathenjwa \& Maharaj, 2012) some clients stated that they did not like using condoms as seen in this comment, "I come here for entertainment, if I do not get satisfaction why should I come here and spend money?" (Ghimire et al. 2011, p.5). Bruce et al., (2011) cited the main reasons for not using condoms as clients' dislike (40\%), trust (35\%) and inability to negotiate by the sex worker (22\%). Some participants felt that the use of condoms was inconvenient and took the enjoyment and fun out of sex (Bruce et al., 2011).

\section{The Female Condom}

A few studies looked at the female condom and how this could be utilised by sex workers to allow them to have agency and take control in situations where male condoms may not be appropriate or available. While the female condom did allow a level of control, many participants did not recommend them due to discomfort (Bailey \& Figueroa 2018). Responses across studies to the female condom were varied, with some women preferring it to the male condom as it gave them control and autonomy and allowed them to initiate its use, often without the detection of uncooperative clients as it could be inserted before sex and just needed practice in the initial stages to become used to (Mathenjwa \& Maharaj, 2012). Some participants explained how the male condom was dependent on the client and they would often come up with excuses to not use one so the female condom was a good option and could be used when male clients offered more money for sex without a condom (Mathenjwa \& Maharaj, 2012). Many FSWs had used the female condom with drunk clients to protect themselves. Several of the studies echoed this sentiment: "Drunk clients are nothing, it is up to me, they follow me, the female condom is very thing and it seems like they wear nothing" (Busza \& Baker, 2004, p.514). The female condom could also not be manipulated or tampered with by clients and were found to be less likely to cause an allergic reaction (Mathenjwa \& Maharaj, 2012).

Some participants preferred the female condom as it did not require a prescription to access and was easier for younger FSWs to acquire although was often limited in terms of accessibility which often meant some FSWs would use one female condom across intercourse with multiple partners (Mathenjwa \& Maharaj, 2012). Female condoms were also found to be easier to discuss with long-term partners in terms of contraception rather than disease prevention 
(Mathenjwa \& Maharaj, 2012). There was some stigma around female condoms by male clients who had not been exposed to them previously (Busza \& Baker, 2004).

\section{Theme 4: Violence/Abuse}

Violence and abuse were prominent themes across multiple studies. Assault from both clients and law enforcement was quite common, especially in settings where sex work is criminalized.

\section{Assault from clients}

Sixteen studies cited violence and abuse as factors in inconsistent condom use with regards to commercial sex (Cheng 2010; Bailey 2018; Ghimire 2011; Maher 2013; Mathenjwa 2012; Urada 2012; Bharat 2013; Bui 2013; Rwema 2019; Decker 2019; Grosso 2015; Shannon 2009; Darbinyan 2011; Alam 2013; Choi 2007; Cusick 1998). Some FSWs cited the fear of violence and sexual assault as a common reason for not using condoms, stating that some clients forcibly prevent the use of a condom (Maher et al., 2013). Drunken clients also cause issues when the FSW tries to negotiate condom use, as they are often too drunk to comply and often threaten violence (Maher et al., 2013). In a 2019 study, physical violence was significantly associated with lower consistent condom use (aPR: $0.71,95 \% \mathrm{CI}=0.52$ to 0.98 ) (Rwema et al., 2019). Similar results were seen in a 2015 study, with verbal and physical harassment being significantly associated with inconsistent condom use (OR: 0.26, 95\% CI= 0.11-0.62) (Grosso et al., 2015).

Many of the women in these studies were aware of the threat of violence from potential partners and exhibited solidarity in protecting themselves, stating that many FSWs carry a weapon such as scissors, knives or pepper spray (Bailey \& Figueroa, 2018). Refusing a client who was unwilling to use condoms was seen as the path of least resistance, whereas trying to convince a client to use condoms was seen as not worth pursuing due to the fear of violence or abuse (Bharat, Mahapatra, Roy \& Saggurti, 2013).

In a 2009 study of 205 FSWs, 25\% of the women reported having been pressured by a client to not use a condom (Shannon et al., 2009). In one study, $30 \%$ of FSW reported sexual abuse and $17.5 \%$ reported physical abuse with the most common perpetrators of sexual abuse being clients $(50.9 \%)$ and stable partners $(28.8 \%)$, while clients were also the most common perpetrators of physical abuse (57\%), followed by stable partners $(22 \%)$, other sex workers $(19 \%)$ and police officers (6\%) (Rwema et al., 2019). Another study found similar results, with $34.8 \%$ of FSWs reporting client-perpetrated violence (Decker et al., 2019). Interestingly, Decker et al., (2019) found that client perpetrated violence was more common among FSWs who had recently experienced intimate partner violence (IPV) $(62.5 \%, \mathrm{P}<0.001)$. This study also concluded that inconsistent condom use in commercial settings was influenced by coercive condom negotiation driven by client intoxication and violence (Decker et al., 2019). Many FSWs perceived that asking a client to use a condom would result in the client becoming violent, with many of these participants reporting prior abuse from clients (Darbinyan, Lang, Diclemente, Joseph \& Markosyan, 2011). FSWs who have experienced prior abuse may be less likely to attempt condom use due to fear of losing a client or facing abuse (Darbinyan et al. 2011). Reports of rape were also prevalent in situations where FSWs attempted to enforce condom use (Cusick, 1998; Choi \& Holroyd, 2007). Violence and rape have been cited as methods to achieve sex without a condom when the FSW does not agree to condomless sex (Cusick, 1998).

\section{Theme 5: Location and support}

The locations of commercial sex transactions and the support from managers, gatekeepers and other facilitators have been shown to have a significant impact on condom use in a commercial setting. Having condoms available on the premises may encourage clients and FSWs to use them, however, for street based sex workers, condoms may not be easily accessible.

\section{Support from managers/gatekeepers}

Some establishments such as brothels may supply condoms on site or have a supportive environment in which management staff or gatekeepers encourage condom use. Wang and colleagues, (2009) found that perceived establishment-level support by FSWs was positively associated with condom use. Higher levels of perceived support for condom use were reported by FSWs with female gatekeepers when compared to FSWs with male or mixed-gender gatekeepers (Yang et al., 2005). Yang et al., (2005) found that more than half of the FSWs included in the study reported no discussion of condom use with their gatekeepers, and $90 \%$ reporting that they had never received a free condom from their gatekeeper, with only $8 \%$ of FSWs reporting that their gatekeeper required them to use condoms with clients and $14 \%$ reporting that refusing to has sex with a client who had refused to use a condom was allowed (Yang et al., 2005). Among the participants of this study who perceived medium to high levels of support by their gatekeepers, 88\% of FSWs had reported having discussions with clients in regards to condom use, whereas only $62 \%$ of FSWs who perceived a low-level of support from their gatekeepers had discussed condom use with clients (Yang et al., 2005). While many FSWs reported that they felt supported in the workplace, perceived support by gatekeepers in relation to condom use was low (Yang et al., 2005). While support from managers was an important factor in consistent condom use, there was so significant 
association with perceived support and correct use on condoms (Yang et al., 2005).

\section{Condoms available on the premises}

Some establishments claimed to supply or sell condoms, however, on observation by research staff, no condoms were available at these establishments as claimed by the owners (Ghimire et al. 2011), whilst in another study, $48 \%$ of establishments had condom use policies in place and 55\% had condoms readily available in the workplace (Urada et al. 2012). Urada et al. (2012) found that having sex without a condom because none were available in the workplace was associated with not negotiating condom use with clients.

\section{Location/setting of sex work}

While establishments such as brothels can offer support and safety to staff, street-based FSWs are often faced with situations that can make condom use difficult. Some street-based sex workers appeared to be more vulnerable to violence and forced non-use of condoms (Maher et al., 2013). In a 2013 study, 20.1\% of street-based FSWs cited the unavailability of condoms (Alam et al., 2013). Some studies found that low-tier or lower SES FSWs were more likely to be street-based and self-employed (Choi \& Holroyd, 2007). In Choi \& Holroyd's (2007) study, consistent condom use among street-based FSWS was low; "If we do ten clients a day, may be only one or two use condoms" (Choi \& Holroyd, 2007, p.494).

\section{Theme 6: Knowledge and perceptions of risk}

Knowledge of the potential risks of forgoing condom use and perceived risk of contracting STIs and HIV was also a common factor in determining condom use, as well as misinformation surrounding the prevention and transmission of STIs. Correct use of condoms by both male clients and FSWs was a factor in predicting consistent condom use.

\section{Sexual health knowledge}

Cheng \& Mak (2010) found that sexual health knowledge was not significantly related to inconsistent condom use. In a 2011 study in which all FSW participants had heard of HIV/AIDS and 98\% were aware of transmission through sexual contact, with $75 \%$ of participants knowing that condom use was the most effective means of preventing HIV, condom use with clients was 'sometimes' 79\% (95\% CI: 73-85), 'never' 15\% (95\% CI: 10-21), and 'always' 6\% (95\% CI: 3-11) (Bruce et al., 2011). This study found that participants had a very basic knowledge of STIs, with very few citing Chlamydia trachomatis and Trichomonas vaginalis as potential risks (Bruce et al., 2011). Wang et al., (2009) also found that there was not a significant direct effect of sexual health knowledge on condom use skills and perceived susceptibility and severity of STIs/HIV. A contrast in these results is seen in Kamal et al. (2015) study in Bangladesh, which suggested that HIV/AIDS related knowledge was the most significant factor that influenced FSWs to suggest condom use to a client. This study also found that condom use was more consistent among FSWs with a higher level of education (Kamal et al. 2015).

Misinformation or a lack of knowledge on how STIs and HIV are spread and contracted appeared in a number of studies. Multiple participants in a 2017 Jamaican study divulged lapses in condom use on assessment of whether a client was disease-free, based on his wealth and aesthetics as shown in the extract below (Bailey \& Figueroa, 2018).

"I use [a] condom with most of the clients, almost all of them; but you really have some very rich man who come with even their HIV results to show you and from you look how they keep themselves neat and clean and smell expensive you know they don’t have any disease". (Bailey \& Figueroa, 2018, p.538)

Misinformation surrounding treatment of STIs and HIV was apparent, with some FSWs stating that washing genitals with antiseptic household cleaner, showering after intercourse or getting married would prevent contracting HIV (Bruce et al., 2011; Zhang et al., 2011). Darbinyan et al. (2001) found that FSWs not using douching as a method to prevent STI/HIV transmission were 4.83 times ( $\mathrm{p}=0.04 ; 95 \%$ CI: $1.04-22.42$ ) more likely to consistently use condoms with clients. Examining the client's genitals prior to intercourse was another strategy used in an attempt to prevent disease among FSWs (Choi \& Holroyd, 2007).

\section{Correct condom use}

An association between condom use self-efficacy and the proper use of condoms was found among FSWs (Zhao et al. 2008). Some participants stated that they were instructed on correct condom use when joining the commercial sex industry and cautioned to use them consistently by peers (Bailey \& Figueroa, 2018). Sometimes the owners of establishments such as massage parlours would educate FSWs on correct condom use, while peer educators were also responsible for instructing peers on correct use (Ghimire et al. 2011). Exposure to interventions or programmes promoting consistent condom use was found to significantly increase condom use, with an adjusted odds ratio of FSWs exposed to the programme being more than double that of the unexposed participants (Blankenship, West, Kershaw \& Biradavolu, 
2008).

\section{Theme 7: Perceptions and Attitudes of Male clients}

The attitudes and beliefs of male clients had a strong impact on the use of condoms in a commercial setting. Poor knowledge of potential STIs, greater than four commercial sex visits, low self-efficacy, less positive social norms and greater alcohol impaired decision making were all factors associated with inconsistent condom use (Wee, Barrett, Lian, Jayabasakar \& Chan, 2004).

\section{Perceived risk and knowledge}

Perceived risk of contracting an STI or HIV was a significant supportive factor of condom use while low perceived risk of infection was found to have a negative impact on condom use (Fauk et al., 2018).

Misinformation or a lack of knowledge about STI's also had a negative impact on condom use, with some male clients stating that if the sex worker looked physically healthy, the client himself was healthy or he had only had intercourse a few times with a sex worker then he could not contract any disease or infection (Fauk et al., 2018). Increased knowledge led to higher consistent condom use as seen in the following quote,

"I just recently started to learn about HIV/AIDS, and now I know a little bit about the means of HIV transmission but before that I did not know, so I did not use condoms at all and I did not think of any risk". (Fauk et al., 2018, p.669)

Negative attitudes surrounding condoms such as discomfort, high prices, less sexual pleasure and reduced satisfaction or sensation were cited by male clients as reasons for not using condoms (Fauk et al., 2018). Inconsistent condom users were also less likely to have heard of STIs such as Hepatitis B or herpes (Wee et al. 2004).

\section{Correct condom use}

Not knowing how to correctly use condoms was also an important factor in their use, while confidence in being able to use condoms correctly was predictive of consistent use, many clients who used condoms inconsistently or not at all stated they did not know how to use them (Fauk et al., 2018; Wee et al. 2004). Male clients who used condoms inconsistently were found to score significantly lower on self-efficacy scoring (Wee et al. 2004).

Higher self-efficacy for condom use was related to positive outcome expectancies for negotiation of safe sex, lower sexual sensation seeking scores and higher social support (Volkmann et al., 2014). For some clients condom use was the norm and they would supply their own for commercial encounters (Bailey \& Figueroa, 2018). One study stated that it was mainly foreign clients that would request or expect to use a condom whereas native clients were not as cautious when it came to commercial sexual encounters (Ghimire et al. 2011).

\section{Social Attitudes and Norms}

Peer disapproval of condom use was a significant risk factor of inconsistent condom use among male clients (Yang et al. 2016). Visiting a commercial sex venue such as a brothel was also common among business clients, with many male respondents stating that they often felt obliged to entertain clients in this way and stated that if the potential business client was openly against condom use, the respondent was also less likely to use condoms due to social pressures (Wee et al. 2004). Some male respondents who had cited previous inconsistent condom use with FSWs were also more likely to forget to use a condom when consuming alcohol, which was common in social situations with groups of men who may visit a brothel (Wee et al. 2004). Some women also reported clients purposefully putting holes in condoms in order to put the health of the FSW in danger (Mathenjwa \& Maharaj, 2012).

\section{Theme 8: Drug and alcohol use}

Thirteen of the included studies cited drug and alcohol use as a factor of inconsistent condom use in commercial sex (Maher 2013; Bui 2013;Jung 2013; Munoz; Decker; Barrington; Zhang 2011; Shannon 2009; Grayman 2005; Alam 2013; Choi 2007; Thorpe 1997; Cusick 1998). Drug and alcohol use among both FSWs and male clients was found to negatively affect condom use.

\section{Substance and alcohol use among FSWs}

A number of studies cited the intoxication of the FSW as a factor in inconsistent condom use (Bailey 2018; Maher 2013; Urada 2012; Bruce 2011; Munoz 2010; Zhang 2011; Alam 2013; Cusick 1998). One study found that substance abuse had a negative impact on the ability of the sex worker to negotiate condom use more than other individual factors such as alcohol use, depression and age, finding that methamphetamine use in particular was quite high (18\% among FSWs using drugs or alcohol) (Urada et al. 2012). Another study found that the majority of FSWs used marijuana, alcohol and homemade stimulants, with almost all of these women stating that they had sex with clients under the influences of one or multiple substances but could not identify a link with HIV risk (Bruce et al., 2011). This contrasts with Jung (2013) 
who found that $74.1 \%$ of FSW responded "never" to using alcohol whilst engaging in sex with a commercial client, while $78 \%$ of male clients responded "often" or "usually".

\section{Client intoxication}

Client intoxication was also a common theme across studies that influenced condom use in transactional sex. Many FSWs identified alcohol intoxication as being prevalent among nearly all clients, especially when the transaction took place in establishments that sold alcohol such as bars and restaurants (Maher et al., 2013). Client intoxication often made condom negotiation by the FSW difficult, and strategies that were normally used such as sweet talk and appealing to the client's health were less effective (Maher et al., 2013). Intoxication of the client was found to have a negative effect on consistent condom use, whereas intoxication of the FSW was more likely to have an effect on non-consistent condom use with a non-paying partner, with one study finding that substance abuse by the FSW was not the influencing factor in condom use and was rather the partner type (Decker et al., 2019). Barrington et al., (2009) found similar results for male client intoxication, stating that male clients who drank less than a few times per week were almost three times more likely to consistently use condoms when compared with clients who consumed alcohol at least a few times per week (OR 2.77, 95\% CI = 1.28-6.02). In a 2005 study of FSWs, only $0.5 \%$ of participants had reported Intravenous drug use (IVDU), however 5\% of FSWs reported ever having a client who was an IVDU and $1.3 \%$ of these women knew that their partners were IVDU (Grayman et al., 2005).

\section{Theme 9: Criminalisation}

Criminalisation was another common theme when looking at barriers to consistent condom use. Many FSWs feared being caught by police and managers or gatekeepers were less likely to support and encourage condom use in settings were sex work was illegal.

\section{Police violence and harassment}

In countries where sex work was illegal, the main focus of the FSW was often to avoid detection or prosecution from law enforcement. Ghimire and colleagues, (2011) found that many of the FSWs involved in the study feared their identity as sex workers being uncovered by police and would not carry condoms on their person, as this would allow them to be charged by police.

Peer-educators were also subject to police violence and harassment after being caught with condoms despite having identity cards, which, in theory should protect them from police violence (Ghimire et al. 2011). The following quote from a peer-educator describes the violence she experienced after being found with condoms,

"The police took me into custody for a couple of months after they found condoms with me. They beat me up with a stick. I had many bruises on my body. At one point I lost my capacity to stand. They also stole all my money from my purse". (Ghimire et al. 2011, p.6)

For these women, the fear of police violence or prosecution from being caught with condoms outweighed the fear of potential health risks. The following quote was described as a typical answer to the question "do you keep condoms in your possession?" and illustrates the intensity of this fear and the implications of being found with condoms by police,

"No I don't... if the police checks my bags searching for a bomb during war, it is sure he will take me to jail. For them both bomb and condoms are similar, both are illegal". (Ghimire et al. 2011, p.6)

Rwema et al., (2019) had similar findings in Senegal, with 26\% of FSWs in the study reporting to avoid carrying condoms on their person due to the fear of police, with $19.5 \%$ having seen a police officer destroy or confiscate condoms carried by themselves or another FSW. FSWs were found to work in unfamiliar locations to avoid detection by police and also rushing negotiations with clients to avoid police (Decker et al., 2019). In locations where sex work is illegal, street-based FSWs are more prone to police harassment when compared with FSWs working in establishments such as brothels and clubs (Choi \& Holroyd, 2007). Due to the illegal status of sex work in China, many FSWs who experience violence or assault do not report crimes to the police and are thus more vulnerable to attacks from clients or law enforcement (Choi \& Holroyd, 2007).

\section{Support from managers/gatekeepers}

Yang et al., (2005) found that a lack of perceived support from gatekeepers may also have a negative effect on condom use, as commercial sex is illegal in China. Carrying or keeping condoms in a work setting such as a brothel has been used to prosecute FSWs and establishments and for this reason condom use may not be encouraged by gatekeepers in countries where sex work is illegal (Yang et al., 2005).

While sex work is legal in Canada, the laws have been described as contradictory, meaning that the environment in which FSWs work is highly prohibitive (Shannon et al. 2009). Women who worked away from main streets to avoid 
persecution from police were three times more likely to be pressured into unprotected sex with a client (Shannon et al. 2009).

\section{Theme 10: Price/payment}

Price and payment were recurrent themes among the included studies. The three main sub-themes that were extracted from the data were gaining financial independence, receiving more money if agreeing to not use a condom and being the sole provider or having dependent family members.

\section{Financial independence}

Bailey \& Figueroa (2018) describes how many of the women in their study had become spontaneously involved in sex work as a way to overcome financial issues and gain financial independence "using the only marketable asset that they possessed - their bodies" (Bailey \& Figueroa 2018). While many of these women had limited ways of earning a substantial income to support themselves and their families, their vulnerable position motivated them to seize an opportunity at economic independence (Bailey \& Figueroa, 2018). In contrast to portraying FSWs as victims of inequity, many women showed individual agency in their decision to enter sex work over other ways of making a living, with many FSWs consciously classifying potential clients based on their ability to pay well and become a source of financial support (Bailey \& Figueroa, 2018). Some studies found that FSW who were better off financially were more likely to report the consistent use of condoms, whereas women who were economically dependent were half as likely to consistently use condoms (Blankenship et al. 2008; Rwema et al., 2019; Munoz et al., 2010). Unprotected sex was reported by FSWs when they were experiencing financial hardship and clients offered to pay significantly higher amounts (Bailey \& Figueroa, 2018).

\section{Higher payment if condoms not used}

Thirteen of the included studies found that the price of sex was considerably higher if condoms were not used (Bailey 2018; Ghimire 2011; Urada 2012; Bruce 2011; Bui 2013; Busza 2004; Rwema 2019; Blankenship 2008; Elmes, 2014; Kamal, 2015; Munoz, 2010; Zhang 2011; Alam 2013). According to Bruce et al., (2011) the majority of FSWs included in their study were compelled to accept higher payment for unsafe sex, with one FSW stating; "Our clients know we are desperate for money, so if they refuse condoms and offer extra cash, it is a proposal I can hardly refuse" (Bruce et al. 2011, p.57). In a study of mainly street-based sex workers, $18 \%$ of participants reported charging more money for unprotected sex and averaged 8 clients per week (Bui et al., 2013). Blankenship et al. (2008) found that $65.5 \%$ of respondents in their study reported being offered a higher price for unprotected sex in the last six months. Elmes et al., (2014) reported that while holding all other variables constant, having unprotected sex with a client increased the mean price by $42.9 \%$. A 2011 study in China found that $13.6 \%$ of FSWs would agree to a client's request to forgo condom use if more money was offered (Zhang et al., 2011). Clients who were not willing to use condoms would often either offer more money or become violent (Alam et al., 2013), putting the FSW in a vulnerable and dangerous position to refuse.

\section{Sole providers}

Many FSWs were the sole providers for their families or had ill relatives that needed to be supported financially. One FSW described how she had accepted a higher price for sex without a condom when her child fell ill (Bailey \& Figueroa, 2018), with another describing risky sexual behaviours to support her family, "The clients told me that he has no HIV. As he paid for sex and I am also paid by my owner to provide services to the clients...If I lose this job I can't survive, and my family has to suffer" (Ghimire et al. 2011, p.4). Choi \& Holroyd (2007) had similar findings, with FSW often facing financial pressure from family crises such as illness of a family member or divorce. Many of the FSWs took pride in their ability to support their families financially (Ghimire et al. 2011) and exhibited individual agency.

\section{Discussion}

A systematic review of 47 studies published in English was conducted to identify the multidimensional structural, social and cultural factors related to condom use in commercial sex work settings. The findings demonstrate that overall, women in control of their sexuality are often considered dangerous and many of the included studies illustrate how female sex workers are often put in situations which compromise their physical and mental well-being and negatively affect condom use.

While many of the included studies were examined under frameworks such as Bandura's Theory of Self-Efficacy (Bandura, 1977) and the Health Belief Model (Strecher, Champion \& Rosenstock, 1997), these frameworks tend to be quite limiting in light of the complex lived experiences and individual and structural-level factors that influence condom use among female sex workers.

Frameworks such as these tend to try and define the reasons condoms are not used in a commercial setting by placing onus on the FSW and denigrating the complexities they face which impact safe sex practices. As mentioned, this review uses a 
feminist approach to interpreting the findings. Doing so acknowledges the multiple factors that influence condom use in the context of female sex work.

The review found that experienced FSWs used high-self efficacy to negotiate with clients to protect their own health and showed that they were good at and enjoyed their work. In contrast to a sex positive lens, abolitionist feminists believe that it is not possible for a sex worker to enjoy or obtain pleasure from sex (Jolly, Cornwall \& Hawkins, 2013). This belief is rooted in gender inequality and the power imbalance between men and women, suggesting that the man, by virtue of his gender has all the power in this dynamic (Jolly et al. 2013). This is refuted by the various accounts of women in the reviewed studies who have shown their power and agency in asserting condom use with clients and choosing to do sex work as an agent of themselves rather than a victim of gender inequality. Feminists, both abolitionist and sex positive, argue that gender inequality means that women who are deemed as sexual are labeled as 'whores' and are a target for social scorn and shame. As the literature shows, it is not the act of exchanging sex for money that damages the physical and psychological integrity of the FSW, but the stigmatization to which they are subjected (Comte, 2013). Stigma is one of the main barriers to accessing health services, with marginalized groups fearing discrimination and rejection from institutions and individuals (Rael, 2015). Some FSWs have described feelings the pressure to fit into society and hide their profession in order to receive the services to which they are entitled (Yingwana, 2018). From a feminist perspective, this social control helps to maintain women's inferiority thus impeding their ability to make effective decisions about their health and wellbeing in a timely manner (Comte, 2013).

Many FSWs express feelings of sexual pleasure that are derived from selling sex, while promoting the sexual agency of women has been suggested to build on the feminist principle of 'erotic justice'. For instance, many women used pleasure in sex work as a way to reclaim their agency, citing sexual pleasure as a feminist choice (Yingwana, 2018). Some women in sex work express that being a sex worker is what makes them a feminist by doing the opposite of what society suggests women should be like or how they should behave (Yingwana, 2018).

These results also show how women have a range of commodities and are adaptive, mixing their work and life identities as seen in relationships that transition from strictly commercial to an emotional connection. The fluid transition between commercial partner and special regular client shows how the line between work and life can often be blurred for women working in the commercial sex industry. It also allows us to understand that for these women, their body is not the only commodity that they are offering, with emotional support or connection sometimes being as valuable to the client as the sex itself. For instance, the women who formed an emotional connection with clients and ceased condom use as a result of a relationship transformation refute Bailey and Figueroa's (2018) claim that all a women has to offer is her body.

While many of these women have adapted to the potentially violent environment of sex work, the prevalence of violence in these studies links back to the idea of women in control of their sexuality being seen as dangerous. Many of these women attempted to take control of their own health by suggesting the use of condoms, however, they were faced with violence from clients who put their own needs of satisfaction or pleasure above the health of the FSW. Feminist scholars suggest that this dynamic reinforces the importance of male sexual pleasure and the subjugation of women sexual or safety needs (Comte, 2013). The assault of peer educators by police was also indicative of the volatile and patriarchal environments that some women worked in. Some FSWs chose to be peer educators, in order to share their lived experiences and provide support and education to other women but were dissuaded by being subject to harassment and violence by law enforcement. This is an example of how violence is used as a method to keep women down and prevent them from educating each other, becoming a collective and achieving solidarity within their profession.

Supportive workplace environments were conducive to more consistent condom use. While environments such a brothels and massage parlours often allowed for collective agency, certain environments, such as the streets, were more patriarchal. Street-based FSWs were often self-employed, allowing limited peer support and interaction and making them more vulnerable to clients and police. Social networks are imperative in allowing women to feel supported to take control of their own health and the situations they face with clients, as also seen in the social networks of male clients promoting condom use in their social networks. From the results, it is clear that street-based sex work is considered more dangerous than working in an establishment or brothel, but why is this? These women are not working with weapons or chemicals or anything considered overtly dangerous, but they are working with men. Why is it that these women need to be vigilant in protecting themselves from men in their occupation? These women go to work, much like anyone else in society, yet they face violence, harassment and ostracism every day with little protection. There are no workplace health and safety measures in settings where sex work is illegal, even though it is a legitimate occupation, the evidence illustrates it is often not viewed this way.

Peer education was shown to be an important factor in consistent condom use, and is evident of the positive outcomes of women who are able to educate each other. Potentially dangerous misinformation such as washing the genitals with household cleaning products could be prevented if education and peer support programmes were prioritized within the 
commercial sex industry.

However, despite some women in these studies having a lack of education in regards to sexual health and HIV/STI transmission, they still demonstrated how they were agents of their own health and were adaptive in taking care of themselves, in addition to maximizing their income.

While the narrative of the sex industry often suggests that all women in sex work are uneducated, this is not the case. Many women in sex work have completed high school or higher education, and have had or still hold a professional position in addition to sex work (Comte, 2013). For some women, it is not a lack of skills or survival needs that make sex work appealing, but rather the advantages such as more money, flexible work hours and self-employment (Comte, 2013). When the gender pay gap exists, sex work is attractive as it gives women an opportunity to earn more than they would in other occupations. Sex work is problematized, as there are no structural changes being made to improve women's pay or working conditions, yet women who choose to earn more and improve their lives via sex work are vilified by society. Some feminists would argue that patriarchal systems construct sex work as deviant because women are taking control of their bodies and using them in ways that make them more money than society would normally give them (Comte, 2013). To reap the benefits and avoid the vilification many sex workers try and hide their profession due to fear of ostracism and the whore-stigma causing low self-esteem in many women (Comte, 2013).

While some societies are more patriarchal than others, physical and sexual assault was a common theme across multiple studies. From the results it is clear that women in general, especially sex workers, are viewed as sex objects with less importance or value than their male counterparts. These ideas are not only demonstrated in the experiences and beliefs of male clients, but also the authors several studies. The attitudes of these authors explicitly highlight the problematic and misogynistic views towards women, especially those in the commercial sex industry. It is these views that perpetuate stigma and the de-humanisation of women in sex work. Research with women involved in sex work in Southern Indiawhere the culture is male dominated, have demonstrated that men's power over women, including intimate partner violence, makes FSWs more vulnerable to HIV, stating that interventions challenging normative gender relations are imperative in improving the health of FSWs (Young et al. 2018). The act of purposefully putting holes in condoms in order to infect or put FSWs at risk of diseases suggests some kind of punishment delivered by the client upon the sex worker (and/or women generally) and is an example of the dangerous attitudes expressed by some clients. To reduce the incidence of these types of violations the de-criminalization and de-stigmatization of sex work is suggested as a vehicle for changing chauvinistic constructions of women's sexuality and making society more open to sex work and FSWs (Comte, 2013).

Some women, in urban India for example choose to do sex work, as their options in regards to making a livelihood are relatively limited (Young et al. 2018). The sex trade in India has been defined as a crucial part of the interconnected array of livelihood strategies that low SES women pursue for survival (Young et al. 2018). Sex work needs to be seen as legitimate work and in a labour market that is structured by gender, we need to alter the structural arrangements of the sex industry in order to enable the agency of women in safe sex practice (Young et al. 2018). Depending on the cultural context, many women in sex work can earn substantially higher incomes than they would if they were involved in other gendered alternatives such as low-skill construction, factory, domestic or agricultural work, however, the less dependence that women place on their income from the sex trade, the higher their capacity to practice safer sex (Young et al. 2018). There is a social conundrum here in that women who are in control of their lives and are reaping benefits from their work are considered problematic, while women who are forced into sex work by means of trafficking do not face the same stigma as they had no agency in becoming involved in the sex industry.

While financial independence increases feelings of control in commercial sex encounters, structural factors also play an important role in the amount of power or control felt by sex workers. In Finland, the social welfare system ensures a good basic income for everyone and thus allows sex workers to reject clients who may insist on not using condoms, or become violent or aggressive (Jolly et al. 2013). This same freedom cannot be experienced by FSWs working in settings where the social welfare system is inadequate or non-existent such as the United States or developing countries (Jolly et al. 2013). The social and legal non-recognition of sex work as a legitimate occupation makes it difficult to obtain working conditions that are in line with the legal minimum norms for other occupations, making it impossible for FSWs to negotiate their working conditions (Comte, 2013).

Criminalisation of sex work has been shown to be largely ineffective and harmful to the health and safety of FSWs. Criminalisation has not reduced the supply nor the demand for sex work but has rather misspent government funds and exacerbated human rights abuses against sex workers (Mgbako et al., 2013). The South African government alone spends approximately $\$ 1.6$ million per year to police and prosecute sex work, with convictions resulting from prosecution of sex work being significantly higher than serious crime convictions such as aggravated robbery and rape (Mgbako et al., 2013). It has been posited that it is not sex work in itself that causes human suffering, but rather the criminalization of sex work 
that is harmful to those involved (Comte, 2013). At the end of the $18^{\text {th }}$ century, while there was a political discourse of citizenship equality, the masculine and them feminine were divided into public and private domains in order to maintain the patriarchal system and dichotomise women as either 'mothers' or 'sexually desiring women' (Comte, 2013). The fear of being caught by police is prominent, as shown in the comparison of being caught with condoms, to being caught with a bomb.

The decriminalization of sex work in New Zealand has illustrated the positive impact that it has had on the health of FSWs (Mgbako et al., 2013). The effects of changing legislation has extensive positive outcomes for sex workers including the rights to refuse clients and certain practices, improved working conditions, improved access to healthcare services, increased condom use, improved relations with police and seeking the help of law enforcement when required and increased confidence and well-being (Mgbako et al., 2013). In addition to this, de-criminalization also did not lead to an increase in sex trafficking, especially underage females, or the number of sex workers as suggested by abolitionist feminists (Mgbako et al., 2013). Post de-criminalization in New Zealand, 77\% of FSWs who were surveyed felt that police officers were concerned for their safety and that their attitudes had change, going from 'prosecutors to protectors' (Mgbako et al., 2013).

From a sex positive feminist point of view, patriarchy is seen as the cause of the sexual repression of women, where women's sexuality is controlled and seen only for is reproductive value (Comte, 2013). The current legislation and attitudes towards women ostracize anyone who does not conform to the traditional monogamous heterosexual sexuality (Comte, 2013). These views are damaging to all women (and all genders), especially FSWs who do not fit the traditional role of monogamous heterosexual female. The perpetuation of these views in society today may explain the violence, harassment and stigma that female sex workers are facing from clients, police and society as a whole. This is a double standard, as men are encouraged to openly express their sexuality, as evident in some of the studies which described the visiting of brothels in groups of friends or even business associates as a bonding exercise, where condom use was often discouraged (Wee et al. 2004; Yang et al. 2016).

Although the social actions of men and women in the context of sex work tend to conform to typical structures of gender, these results are a combination of individual actions reproducing social structure and social structures that are shaped by individual actions (Young et al. 2018). In order to understand how structural factors influence condom use in the sex industry, sexual risk behaviours need to be reframed as gendered sexual practices (Young et al. 2018). Young et al. (2018) suggests that "interconnected structures of labour markets and patriarchal domination, in addition to emerging patterns of resistance to sexual violence and social stigma constitute the social context in which women in sex work engage in gendered sexual practices" (Young et al. 2018, p. 863). Social systems are inherently patriarchal and contribute to the difficulties that women in sex work face every day. Taking these contextual factors into consideration need to be utilized to analyse structural supports for the capacity of FSWs in order to increase condom use and overall sexual health by reshaping interactional expectations of social sexual practice (Young et al. 2018).

\section{Limitations}

The majority of the studies included in this review were from Asian and African nations, with only three studies from Western countries (UK, USA and Canada). The studies from these countries were relatively outdated and there was a notable difference in the language used (i.e., the term "prostitute") which are rarely used in more recent studies. There is a clear dearth of research from Western countries and therefore the generalizability of the findings in this systematic review is limited.

A lack of theory in many of the papers used in this systematic review is also evident. In addition, many of the studies that used theory drew on Bandura's theory of self-efficacy, and did not take important structural and cultural contextual factors into consideration in their analysis. While some theories were used to help guide the research, they were limited in their ability to incorporate structural and social determinants in relation to condom use in female sex work.

There was an unequal balance of qualitative (7) versus quantitative (40) studies, which may have affected some of the findings. Including more qualitative studies may have better represented the experiences and perspectives of women in sex work.

\section{Implications}

Theory

Feminist theory helps us to explore the experiences of the female sex worker through a particular lens that highlights the strength of women. Interpreting these findings through this lens has highlighted a need for systemic change in the way women and FSWs in particular are viewed. Theories used as a guide should be multi-faceted and include structural and social determinants. Feminist theory is important in the context of sex work as it allows us to examine the factors that influence condom use whilst taking other structural and social influences into consideration. 


\section{Education/Practice}

Educational interventions based on increasing sexual health knowledge, while important, may not be the most effective means of increasing condom use among FSWs. The results have shown that adequate health knowledge is not the most important determinant in consistent condom use and interventions should also focus their efforts towards empowering FSWs and managers as a collective.

The attitudes of male clients also need to be targeted in interventions to ensure that condoms are being used appropriately, but also the health of the female partner is seen as more important than the desires of the client. Underlying sexism, especially in more patriarchal societies is a challenge that also needs to be addressed by interventions.

While society as a whole can be educated, it is difficult for social change to occur without structural change. Structural barriers in terms of policy and legislation inhibit the rights of sex workers and exacerbate social marginalization and stigma. Female sex workers, especially in more male dominant societies and cultures. will be looked down upon and deemed as less until legislative change occurs. The way society as a whole conceives sex work, especially under a patriarchal framework, needs to be reframed and sex work needs to be seen as a legitimate occupation.

\section{Future Research}

Many of the studies included in this systematic review tend to focus on what is going wrong in regards to condom use in sex work. There was an apparent lack of recent studies from Western/European countries where decriminalization has been successful in increasing condom use in a commercial setting and improving the health and safety of women in the sex industry. Investigating the experiences of FSWs and clients in these countries and comparing the findings to those in this systematic review may be imperative in improving the health and safety of FSWs globally.

\section{Conclusion}

This systematic review examined 47 studies on factors influencing condom use among female sex workers. A wide range of qualitative, quantitative and mixed methods studies were synthesized and appraised using a feminist framework. While a range of factors were identified to influence condom use in the commercial sex industry, many of these were structural determinants which oppressed the human rights and safety of women in sex work. In order to improve the public health of FSWs, this review has illustrated that we need to look at the bigger picture of the systematic oppression of women in sex work.

The sexual oppression of women cannot be undone by pushing men to conform to the same repressive sexual norms that presently control women's sexuality, as suggested by abolitionist feminists (Comte, 2013). This would only serve to further perpetuate the sexual repression of women and the ostracism and marginalization of those who express sexuality outside of the social and structural constraints they are put in (Comte, 2013). It is only by questioning these social norms and decriminalizing sex work to allow it to be seen as a legitimate occupation that female sex workers will be protected from physical violence, assault and psychological damage (Comte, 2013). The existing stigma and prejudice towards female sex workers must be fought and policy makers must recognize that they have the same rights to police protection, social respect and safe working conditions as the rest of society (Comte, 2013).

\section{References}

Ahmed, A. (2011). Feminism, Power and Sex Work in the context of HIV/AIDS: Consequences for women's health. Harvard Journal of Law \& Gender, 34(1), 225-258.

Alam, N., Chowdhury, M., Mridha, M., Ahmed, A., Reichenbach, L., Streatfield, P., \& Azim, T. (2013). Factors associated with condom use negotiation by female sex workers in Bangladesh. International Journal of STD \& AIDS, 24(10), 813-821. https://doi.org/10.1177/0956462413486452

Bailey, A., \& Figueroa, J. (2018). Agency, lapse in condom use and relationship intimacy among female sex workers in Jamaica. Culture, Health \& Sexuality, 20(5), 531-544. https://doi.org/10.1080/13691058.2017.1360944

Bandura, A. (1977). Self-efficacy: Toward a unifying theory of behavioral change. Psychol Rev, 84(2), 191-215. https://doi.org/10.1037/0033-295X.84.2.191

Barrington, C., Latkin, C., Sweat, M., Moreno, L., Ellen, J., \& Kerrigan, D. (2009). Talking the talk, walking the walk: Social network norms, communication patterns, and condom use among the male partners of female sex workers in La Romana, Dominican Republic. Soc Sci Med, 68(11), 2037-2044. https://doi.org/10.1016/j.socscimed.2009.03.009

Bharat, S., Mahapatra, B., Roy, S., \& Saggurti, N. (2013). Are Female Sex Workers able to Negotiate Condom Use with Male Clients? The Case of Mobile FSWs in Four High HIV Prevalence Sates of India. PLOS One, 8(6), 1-8. 
https://doi.org/10.1371/journal.pone.0068043

Blankenship, K., West, B., Kershaw, T., \& Biradavolu, M. (2008). Power, community mobilization, and condom use practices among female sex workers in Andhra Pradesh, India. AIDS, 22, S109-S116. https://doi.org/10.1097/01.aids.0000343769.92949.dd

Bruce, E., Bauai, L., Sapuri, M., Kaldor, J., Fairley, C., \& Keogh, L. (2011). HIV knowledge, risk perception, and safer sex practices among female sex workers in Port Moresby, Papua New Guinea. International Journal of Women's Health, 3, 53-61. https://doi.org/10.2147/IJWH.S14669

Bui, T., Markham, C., Tran, L., Palmer Beasly, R., \& Ross, M. (2013). Condom Negotiation and Use Among Female Sex Workers in Phnom Penh, Cambodia. AIDS Behav, 17, 612-622. https://doi.org/10.1007/s10461-012-0369-9

Busza, J., \& Baker, S. (2004). Protection and participation: an interactive programme introducing the female condom to migrant sex workers in Cambodia. AIDS CARE, 16(4), 507-518. https://doi.org/10.1080/09540120410001683457

Cheng, S., \& Mak, W. (2010). Contextual influences on safer sex negotiation among female sex workers (FSWs) in Hong Kong: the role of non-governmental organizations (NGOs), FSWs' managers, and clients. AIDS CARE, 22(5), 606-613. https://doi.org/10.1080/09540120903311441

Choi, S., \& Holroyd, E. (2007). The influence of power, poverty and agency in the negotiation of condom use for female sex workers in mainland China. Culture, Health \& Sexuality, 9(5), 489-503 https://doi.org/10.1080/13691050701220446

Clarkson, F. (1939). History of Prostitution. The Canadian Medical Association Journal, 41(3), 296-301.

Comte, J. (2014). Decriminalization of Sex Work: Feminist Discourses in Light of Research. Sexuality \& Culture, 18(1), 196-217. https://doi.org/10.1007/s12119-013-9174-5

Cusick, L. (1998). Non-use of condoms by prostitute women, AIDS CARE, 10(2), 133-146. https://doi.org/10.1080/09540129850124406

Darbinyan, N., Lang, D., Diclemente, R., Joseph, J., \& Markosyan, K. (2011). Application of Condoms on Male Clients by Female Sex Workers in Yerevan, Armenia: Prevalence and Correlates. J . Biosoc. Sci, 43, 575-585. https://doi.org/10.1017/S0021932011000204

Decker, M., Park, J., Allen, S., Silberzahn, B., Footer, K., Huettner, S., Galai, N., \& Sherman, S. (2019). Inconsistent Condom Use Among Female Sex Workers: Partner-specific Influences of Substance Use, Violence, and Condom Coercion. AIDS and Behavior. https://doi.org/10.1007/s10461-019-02569-7

Elmes, J., Nhongo, K., Ward, H., Hallett, T., Nyamukapa, C., White, P., \& Gregson, S. (2014). The Price of Sex: Condom Use and the Determinants of the Price of Sex Among Female Sex Workers in Eastern Zimbabwe. The Journal of Infectious Diseases, 210, 569-578. https://doi.org/10.1093/infdis/jiu493

Fauk, N., Kustanti, C., Liana, D., Indriyawati, N., Crutzen, R., \& Mwanr, L. (2018). Perceptions of Determinants of Condom Use Behaviors Among Male Clients of Female Sex Workers in Indonesia: A Qualitative Inquiry. American Journal of Men's Health, 12(4), 666-675. https://doi.org/10.1177/1557988318756132

Ford, K., Wirawan, D., Reed, B., Muliawan, P., \& Sutarga, M. (2000). AIDS and STD knowledge, condom use and HIV/STD infection among female sex workers in Bali, Indonesia. AIDS CARE, 12(5), 523-534. https://doi.org/10.1080/095401200750003716

Gavey, N., McPhillips, K., \& Doherty, M. (2001). “If it's not on, it's not on"- or is it? Discursive constraints on women's condom use. Gender \& Society, 15, 917-934. https://doi.org/10.1177/089124301015006008

Ghimire, L., Smith, C., van Teijlingen, E., Dahal, R., \& Luitel, N. (2011). Reasons for non-use of condoms and self-efficacy among female sex workers: A qualitative study in Nepal. BMC Women's Health, 11(42). https://doi.org/10.1186/1472-6874-11-42

Grayman, J., Nhan, D., Huong, P., Jenkins, R., Carey, J., West, G., \& Minh, T. (2005). Factors Associated with HIV Testing, Condom Use, and Sexually Transmitted Infections Among Female Sex Workers in Nha Trang, Vietnam. AIDS and Behavior, 9(1), 41-51. https://doi.org/10.1007/s10461-005-1680-5

Grosso, A., Lei, E., Ketende, S., Peitzmeier, S., Mason, K., Ceesay, N., ... Baral, S. (2015). Correlates of condom use among female sex workers in The Gambia: results of a cross-sectional survey. Peer J. https://doi.org/10.7717/peerj.1076

Hong, Q. N., Pluye, P., Fabregues, S., Bartlett, G., Boardman, F., Cargo, M., ... Vedel, I. Mixed Methods Appraisal Tool (MMAT) version 2018. Registration of Copyright (\#1148552), Canadian Intellectual Property Office, Industry 
Canada.

Jolly, S., Cornwall, A, editor \& Hawkins, K, editor. (2013). Women, Sexuality and the Political Power of Pleasure: Sex, Gender and Empowerment. Zed Books.

Jung, M. (2013). Sexual Behavior and Condom Use among Gay Men, Female Sex Workers, and Their Customers: Evidence from South Korea. PLOS One, 8(6). https://doi.org/10.1371/journal.pone.0066867

Kamal, M., Hassan, C., \& Salikon, R. (2015). Safer Sex Negotiation and Its Association With Condom Use Among Clients of Female Sex Workers in Bangladesh. Asia-Pacific Journal of Public Health, 27(2), NP2410-2422. https://doi.org/10.1177/1010539513510554

Kerrigan, D., Ellen, J., Moreno, L., Rosario, S., Katz, J., Celentano, D., \& Sweat, M. (2002). Environmental-structural factors significantly associated with consistent condom use among female sex workers in the Dominican Republic. AIDS, 17, 415-423. https://doi.org/10.1097/00002030-200302140-00016

Lausevic, D., Begic, S., Terzic, N., Vratnica, Z., Cicic, A., \& Mugosa, B. (2016). Factors associated with inconsistent condom use with clients among female sex workers in Podgorica, Montenegro. Srp Arh Celok Lek, 144(7-8), 424-430. https://doi.org/10.2298/SARH1608424L

Lee, J., Jung, S., Kwon, D., Jung, M., \& Park, B. (2010). Condom Use and Prevalance of Genital Chlamydia trachomatis Among the Korean Female Sex Workers. Epidemiology and Health, 32. https://doi.org/10.4178/epih/e2010008

Ma, Q., Jiang, J., Pan, X., Cai, G., Wang, H., Zhou, X., Jiang, T., \& Chen, L. (2017). Consistent condom use and its correlates among female sex workers at hair salons: a cross-sectional study in Zhejiang province, China. BMC Public Health, 17(910). https://doi.org/10.1186/s12889-017-4891-6

Maher, L., Mooney-Somers, J., Phlong, P., Couture, M., Kien, S., Stein, E., .. Page, K. (2013). Condom negotiation across different relationship types by young women engaged in sex work in Phnom Penh, Cambodia. Glob Public Health, 8(3), 270-283. https://doi.org/10.1080/17441692.2013.767930

Mathenjwa, T., \& Maharaj, P. (2012). 'Female condoms give women greater control': A qualitative assessment of the experiences of commercial sex workers in Swaziland. The European Journal of Contraception \& Reproductive Health Care, 17(5), 383-392. https://doi.org/10.3109/13625187.2012.694147

Mgbako, C., Bass, K., Bundra, E., Jamil, M., Keys, J., \& Melkus, L. (2013). The Case for Decriminalisation of Sex Work in South Africa. Georgetown Journal of International Law, 44(4), 1423-1454.

Moher, D., Liberati, A., Tetzlaff, J., Altmann, D. G., \& P, Group. Preferred reporting items for systematic reviews and meta-analyses: the PRISMA statement. PLoS Medicine. 2009, 6(7), e1000097. https://doi.org/10.1371/journal.pmed.1000097

Munoz, F., Pollini, R., Zuniga, M., Strathdee, S., Lozada, R., Martinez, G., ... Patterson, T. (2010). Condom Access: Associations with Consistent Condom Use Among Female Sex Workers in Two Northern Border Cities of Mexico. AIDS Education and Prevention, 22(5), 455-465. https://doi.org/10.1521/aeap.2010.22.5.455

Pajnik, M. (2012) Reconciling Paradigms of Prostitution through Narration. Drustvena Istrazivanja, $22(2), 257-276$. https://doi.org/10.5559/di.22.2.03

Parcesepe, A., L’Engle, K., Martin, S., Green, S., Suchindran, C., \& Mwarogo, P. (2016). Early sex work initiation and condom use among alcohol-using female sex workers in Mombasa, Kenya: a cross-sectional analysis. Sex Transm Infect, 92, 593-598. https://doi.org/10.1136/sextrans-2016-052549

Qu, S., Liu, W., Choi, K., Li, R., Jiang, D., Zhou, Y., ... Mandel, J. (2002). The Potential for Rapid Sexual Transmission of HIV in China: Sexually Transmitted Diseases and Condom Failure Highly Prevalent among Female Sex Workers. AIDS and Behavior, 6(3), 267-275. https://doi.org/10.1023/A:1019848026130

Rael, C. (2015). Perceptions of Sex Work-Related Stigma in Female Sex Workers from the Dominican Republic: Implications for HIV Interventions. Sexuality \& Culture, 19(4), 674-684. https://doi.org/10.1007/s12119-015-9284-3

Rwema, J., Lyons, C., Ketende, S., Bowring, A., Rao, A., Comins, C., .. Baral, S. (2019). Characterizing the Influence of Structural Determinants of HIV Risk on Consistent Condom Use Among Female Sex Workers in Senegal. J Acquir Immune Defic Syndr, 81, 63-71. https://doi.org/10.1097/QAI.0000000000001991

Saggurti, N., Mishra, R., Proddutoor, L., Tucker, S., Kovvali, D., Parimi, P., \& Wheeler, T. (2013). Community collectivization ands its association with consistent condom use and STI treatment-seeking behaviors among female sex workers and high-risk men who have sex with men/transgenders in Andhra Pradesh, India. AIDS CARE, 25, 
55-66. https://doi.org/10.1080/09540121.2012.749334

Sedyaningsih-Mahamit, \& E. Gortmaker, S. (1999). Determinants of Safer-Sex Behaviors of Brothel Female Commercial Sex Workers in Jakarta, Indonesia. The Journal of Sex Research, 36(2), 190-197. https://doi.org/10.1080/00224499909551984

Shannon, K., Strathdee, S., Shoveller, J., Rusch, M., Kerr, T., \& Tyndall, M. (2009). Structural and Environmental Barriers to Condom Use Negotiation With Clients Among Female Sex Workers: Implications for HIV-Prevention Strategies and Policy. American Journal of Public Health, 99(4), 659-665. https://doi.org/10.2105/AJPH.2007.129858

Strecher, V. J., Champion, V. I., \& Rosenstock, I. M. (1988). Social Learning Theory and the Health Belief Model. Health education quarterly, 15(2), 175-183. https://doi.org/10.1177/109019818801500203

Thomas, H. (2003). Quality assessment tool for quantitative studies. Effective public health practice project Toronto. McMaster University.

Thomas, H., \& Harden, A. (2008). Methods for the thematic synthesis of qualitative research in systematic reviews. BMC Medical Research Methodology, 8(1), 45. https://doi.org/10.1186/1471-2288-8-45

Thorpe, L., Ford, K., Fajans, P., \& Wirawan, D. (1997). Correlates of condom use among female prostitutes and tourist clients in Bali, Indonesia. AIDS CARE, 9(2), 181-197. https://doi.org/10.1080/09540129750125208

Urada, L., Morisky, D., Pimentel-Simbulan, N., Silverman, J., \& Strathdee, S. (2012). Condom Negotiations among Female Sex Workers in the Philippines: Environmental Influences. PLOS One, 7(3). https://doi.org/10.1371/journal.pone.0033282

Vandepitte, J., Lyerla, R., Dallabetta, G., Crabbé, F., Alary, M., \& Buvé, A. (2006). Estimates of the number of female sex workers in different regions of the world. Sex Trans Infect, 82(3), 18-25. https://doi.org/10.1136/sti.2006.020081

Vejella, S., Patel, S., Saggurti, N., \& Prabhakar, P. (2016). Community Collectivization and Consistent Condom Use Among Female Sex Workers in Southern India: Evidence from Two Rounds of Behavioral Tracking Surveys. AiDS Behav, 20, 776-787. https://doi.org/10.1007/s10461-015-1171-2

Volkmann, T., Wagner, K., Strathdee, S., Semple, S., Ompad, D., Chavarin, C., \& Patterson, T. (2014). Correlates of Self-Efficacy for Condom Use Among Male Clients of Female Sex Workers in Tijuana, Mexico. Arch Sex Behav, 43, 719-727. https://doi.org/10.1007/s10508-013-0149-2

Wang, B., Li, X., McGuire, J., Kamali, V., Fang, X., \& Stanton, B. (2009). Understanding the Dynamics of Condom Use Among Female Sex Workers in China. Sexually Transmitted Diseases, 36(3), 134-140. https://doi.org/10.1097/OLQ.0b013e318191721a

Wee, S., Barrett, M., Lian, W., Jayabasakar, T., \& Chan, K. (2004). Determinants of inconsistent condom use with female sex workers among men attending the STD clinic in Singapore. Sex Transm Infect, 80, 310-314. https://doi.org/10.1136/sti.2003.008342

Wiechelt, S., \& Shdaimah, C. (2015). Condoms and Cupcakes: Fostering Autonomy Through Relationships of Care With Women in Prostitution. Journal of Progressive Human Services, 26(2), 166-185. https://doi.org/10.1080/10428232.2015.1018109

Wong, M., Chan, R., Koh, D., \& Wee, S. (1999). Factors Associated With Condom Use for Oral Sex Among Female Brothel-Based Sex Workers in Singapore. Sexually Transmitted Diseases, 27(1), 39-45. https://doi.org/10.1097/00007435-200001000-00008

Yang, H., Li, X., Stanton, B., Fang, X., Zhao, R., Dong, B., ... Hong, Y. (2005). Condom Use Among Female Sex Workers in China: Role of Gatekeepers. Sexually Transmitted Diseases, 32(9), 572-580. https://doi.org/10.1097/01.olq.0000175418.48665.95

Yang, Y., Yang, C., Latkin, C., Luan, R., \& Nelson, K. (2016). Condom Use During Commercial Sex Among Male Clients of Female Sex Workers in Sichuan, China: A Social Cognitive Theory Analysis. AiDS Behav, 20, 2309-2317. https://doi.org/10.1007/s10461-015-1239-Z

Ye, X., Shang, M., Shen, T., Pei, B., Jiang, X., \& Cai, Y. (2012). Social, psychological, and environmental-structural factors determine consistent condom use among rural-to-urban migrant female sex workers in Shanghai, China. BMC Public Health, 12(599). https://doi.org/10.1186/1471-2458-12-599

Yingwana, N. (2018). "We Fit in Society by Force": Sex Work and Feminism in Africa. Meridians: feminism, race, transnationalism, 17(2), 279-295. https://doi.org/10.1215/15366936-7176439 
Young, G., Danner, M., Fort, L., \& Blankenship, K. (2018). Gender and sexual practice in structural context: Condom Use among Women Doing Sex Work in Southern India. Gender \& Society, 32(6), 860-888. https://doi.org/10.1177/0891243218787757

Zhang, H., Liao, M., Nie, X., Pan, R., Wang, C., Ruan, S., ... Jiang, B. (2011). Predictors of consistent condom use based on the Information-Motivation-Behavioral Skills (IMB) model among female sex workers in Jinan, China. BMC Public Health, 11(113). https://doi.org/10.1186/1471-2458-11-113

Zhao, J., Song, F., Ren, S., Wang, Y., Wang, L., Liu, W., ... Sun, Y. (2012). Predictors of Condom Use Behaviors Based on the Health Belief Model (HBM) among Female Sex Workers: A Cross-Sectional Study in Hubei Province, China. PLOS One, 7(11). https://doi.org/10.1371/journal.pone.0049542

Zhao, R., Wang, B., Fang, X., Li, X., \& Stanton, B. (2008). Condom use self-efficacy among female sex workers with steady partners in China. AIDS CARE, 20(7), 782-790. https://doi.org/10.1080/09540120701694030

Zhou, C., Hsieh, E., Rou, K., Tillman, J., Dong, W., Feng, X. Y., ... Wu, Z. (2019). Short-term acceptability of female condom use among low-fee female sex workers in China: a follow-up study. BMC Women's Health, 19(77). https://doi.org/10.1186/s12905-019-0773-7

\section{Copyrights}

Copyright for this article is retained by the author(s), with first publication rights granted to the journal.

This is an open-access article distributed under the terms and conditions of the Creative Commons Attribution license which permits unrestricted use, distribution, and reproduction in any medium, provided the original work is properly cited. 